Behavioral/Systems/Cognitive

\title{
Broadband Coding with Dynamic Synapses
}

\author{
Benjamin Lindner, ${ }^{1}$ Dorian Gangloff, ${ }^{2}$ André Longtin, ${ }^{2,4}$ and John E. Lewis ${ }^{3,4}$ \\ ${ }^{1}$ Max Planck Institute for the Physics of Complex Systems, 01187 Dresden, Germany, and Departments of ${ }^{2}$ Physics and ${ }^{3}$ Biology and ${ }^{4}$ Center for Neural \\ Dynamics, University of Ottawa, Ottawa, Ontario, Canada KIN 6N5
}

Short-term synaptic plasticity (STP) can significantly alter the amplitudes of synaptic responses in ways that depend on presynaptic history. Thus, it is widely assumed that STP acts as a filter for specific patterns of presynaptic inputs, and as a result can play key roles in neuronal information processing. To evaluate this assumption and directly quantify the effects of STP on information transmission, we consider a population of independent synaptic inputs to a model neuron. We show using standard information theoretic approaches that the changes in synaptic response amplitude resulting from STP interact with the related effects on fluctuations in membrane conductance, such that information transmission is broadband (no frequency-dependent filtering occurs), regardless of whether synaptic depression or facilitation dominates. Interestingly, this broadband transmission is preserved in the postsynaptic spike train as long as the postsynaptic neuron's baseline firing rate is relatively high; in contrast, low baseline firing rates lead to STP-dependent effects. Thus, background inputs that control the firing state of a postsynaptic neuron can gate the effects of STP on information transmission.

Key words: coherence; facilitation; information transmission; neural dynamics; short-term plasticity; synaptic depression

\section{Introduction}

Short-term synaptic plasticity (STP) acts on a variety of timescales to change the amplitude of postsynaptic responses to presynaptic spike trains (Abbott and Regehr, 2004; Morrison et al., 2008). The functional roles that STP is thought to play depend on the nature of the presynaptic inputs. STP can enhance the detection of transient inputs, such as presynaptic bursts and abrupt changes in input rate (Abbott et al., 1997; Lisman, 1997; Puccini et al., 2007). In the case of stationary presynaptic inputs (i.e., inputs with constant mean rate), STP is generally thought to lead to high-pass or low-pass filtering (Dittman et al., 2000; Fortune and Rose, 2001; Abbott and Regehr, 2004). This idea is based on the changes in synaptic response amplitude (synaptic gain) during stimulation of presynaptic inputs at different rates (Dittman et al., 2000; Lewis and Maler, 2002; Klyachko and Stevens, 2006): facilitation leads to increases in amplitude with increasing input rate, while short-term depression leads to decreases, suggesting high-pass and low-pass filtering, respectively.

A description of such changes in synaptic amplitude is not sufficient, however, to fully describe information transmission across a synapse. To do this, one must also consider changes in synaptic amplitude in the context of accompanying noise sources (Rieke et al., 1996; Gabbiani and Koch, 1998; Stein et al., 2005). Under natural conditions, synaptic inputs can involve populations of asynchronously firing presynaptic neurons, with the resulting synaptic activity providing a major source of membrane

\footnotetext{
Received Aug. 5, 2008; revised Dec. 15, 2008; accepted Dec. 17, 2008.

This work was supported by Natural Sciences and Engineering Research Council of Canada Discovery grants (A.L., J.E.L.) and an Ontario Premier's Research Excellence Award (A.L.). We thank Matthias Merkel for helpful comments on this manuscript.

Correspondence should be addressed to John E. Lewis, Department of Biology, University of Ottawa, 30 MarieCurie, 0ttawa, Ontario, Canada K1N 6N5. E-mail: jlewis@uottawa.ca. DOI:10.1523/JNEUROSCI.3702-08.2009

Copyright $\odot 2009$ Society for Neuroscience $\quad$ 0270-6474/09/292076-12\$15.00/0
}

voltage fluctuations, or noise (Jacobson et al., 2005). In addition, one must consider how time-dependent synaptic amplitudes and associated fluctuations are affected by time-varying inputs. Finally, the influence of these dynamic synaptic inputs on postsynaptic spike trains must be understood. Fluctuations in membrane voltage near spiking threshold can greatly influence postsynaptic spike trains (Chance et al., 2002; Kuhn et al., 2004; Stein et al., 2005). How these different features interact to influence information transmission across the synapse is not clear.

In this study, we investigate how STP shapes information transmission in a spiking model neuron receiving input from a population of independent synapses using a common formalism to model synaptic plasticity (Abbott et al., 1997; Tsodyks and Markram, 1997; Dittman et al., 2000; Lewis and Maler, 2002; Abbott and Regehr, 2004; Puccini et al., 2007; Mongillo et al., 2008; Morrison et al., 2008). We first characterize the synaptic activity in the context of spontaneous background inputs, and then quantify information transmission of a signal encoded by a rate-modulated input. We show that information transmission across a synapse can be independent of the frequency of this rate-modulated input regardless of whether facilitation or depression dominates. Information transmission is thus broadband, with no STP-dependent filtering. Interestingly, the expression of this effect in a postsynaptic spike train depends on the state of the postsynaptic neuron: broadband coding is preserved when the neuron is firing at moderate to high rates, but STP-dependent filtering occurs when the neuron is firing at low rates. These results suggest the intriguing possibility that the background synaptic inputs shown to be involved in controlling firing rate, response gain, stochastic resonance, and phase locking (French et al., 1972; Knight, 1972; Longtin, 1993; Doiron et al., 2001; Chance et al., 2002; Le Masson et al., 2002; Stein et al., 2005), can also play a more complex role in neural coding by controlling a switch between broadband information transmission and STP-dependent filtering. 


\section{Materials and Methods}

Our overall approach involves well established techniques to model and quantify short-term synaptic plasticity, neuronal firing dynamics, and information transmission (Rieke et al., 1996; Gabbiani and Koch, 1998; Dayan and Abbott, 2001). We summarize the important aspects below and include additional technical details in the Appendix.

FD-modeling formalism and LIF neuron model. In the first part of our study, we consider only spontaneous background synaptic inputs. In this case, a population of inputs $(N=100)$ are described by Poissonian presynaptic spike trains denoted by $\xi_{j}(t)=\Sigma_{i} \delta\left(t-t_{i, j}\right)$, where $t_{i, j}$ is the $i$ th spiking time of the $j$ th presynaptic input and the mean firing rate for each input is $r=\left\langle\xi_{j}(t)\right\rangle$ (chosen to be equal for all synapses and thus independent of $j$ ). Here and in the following, $\langle>$ denotes an ensemble average. The synaptic amplitude due to the $i$ th input spike at the $j$ th synapse is given by the product $F_{i, j} D_{i, j}$, where $F_{j}(t)$ and $D_{j}(t)$ are the facilitation and depression variables of the $j$ th synapse following the dynamics given by the following equations:

$$
\begin{gathered}
\dot{D}_{j}=\frac{1-D_{j}}{\tau_{D}}, t=t_{i, j} \Rightarrow D_{j} \rightarrow D_{j}\left(1-F_{j}\right) \\
\dot{F}_{j}=\frac{F_{0}-F_{j}}{\tau_{F}}, t=t_{i, j} \Rightarrow F_{j} \rightarrow F_{j}+\Delta \\
F_{j}(t)>1 \Rightarrow F_{j}(t) \rightarrow 1 .
\end{gathered}
$$

At the time of an input spike $\left(t=t_{i, j}\right), D$ is first updated (decreased by an amount $\left.F_{i, j} D_{i, j}\right)$; then $F$ is updated by an increment $\Delta$; for the synaptic amplitudes, we take the values at a time $t_{i}^{-}$just before these updates, i.e., $F_{i, j}=F_{j}\left(t_{i}^{-}\right), D_{i, j}=D_{j}\left(t_{i}^{-}\right)$. We have introduced an upper bound for $F$, i.e., $F \leq 1$, to prevent negative values of the update factor of the depression variable. For very low input spike rates, $F \approx F_{0}$ and $D \approx 1$. The $F D$-modulated synaptic input at the $j$ th synapse is $x_{j}(t)=\Sigma_{i} F_{i, j} D_{i, j} \delta(t-$ $t_{i, j}$ ) and the total synaptic input (summed over all $N=100$ synapses) is denoted by $X(t)=\Sigma_{i, j} F_{i, j} D_{i, j} \delta\left(t-t_{i, j}\right)$. The mean amplitude at low input rate can be approximated by the following (see Appendix):

$$
\begin{aligned}
& \left\langle F_{i, j} D_{i, j}\right\rangle \\
= & \frac{2\left[F_{0}+\Delta r \tau_{F}+\frac{1}{2 r}\left(\frac{1}{\tau_{F}}+\frac{1}{\tau_{D}}\right)\right]^{2}-\frac{1}{2 r^{2}}\left(\frac{1}{\tau_{P}}+\frac{1}{\tau_{D}}\right)^{2}-\Delta^{2} r \tau_{F}}{2 r \tau_{D}\left[F_{0}+\Delta r \tau_{F}+\frac{1}{r}\left(\frac{1}{2 \tau_{F}}+\frac{1}{\tau_{D}}\right)\right]^{2}-\tau_{D}\left(\Delta^{2} r^{2} \tau_{F}+\frac{1}{2 r \tau_{F}^{2}}\right)+2 \Delta} .
\end{aligned}
$$

The formula fits the simulation results even at larger rates (compare Fig. $1 B)$.

The postsynaptic conductance due to the total synaptic input $X(t)$ is given in our model by $\dot{G}=-G / \tau+\left(g_{0} / N\right) X(t)$, where $\tau=5 \mathrm{~ms}$ and $g_{0} / N=4 \mathrm{nS}$. This conductance then drives a leaky integrate-and-fire (LIF) model neuron described by $C_{\mathrm{m}} \dot{V}=-g_{\mathrm{L}}\left(V-V_{\mathrm{L}}\right)-G(t)\left(V-V_{\mathrm{E}}\right)$ $+\mu$ and a fire-and-reset rule. Here, $\dot{V}$ represents the time derivative of $V$; $V_{\mathrm{L}}=-70 \mathrm{mV}$ and $V_{\mathrm{E}}=0 \mathrm{mV}$ denote the leak and the excitatory reversal potentials, respectively; $g_{\mathrm{L}}=100 \mathrm{nS}$ is the leak conductance, $C_{\mathrm{m}}=1 \mathrm{nF}$ is the membrane capacitance, and $\mu$ is a constant bias current. The firing threshold is $V_{\text {thr }}=-65 \mathrm{mV}$, and after firing the voltage is reset to $V_{\text {reset }}$ $=-70 \mathrm{mV}$. We denote the LIF output spike train by $y(t)=\Sigma_{k} \delta\left(t-t_{k}\right)$.

Spectral measures of membrane conductance fluctuations. To quantify the membrane fluctuations at different frequencies, we use the power spectrum. Given a certain time series $Z(t)$ (input or output spike train or conductance fluctuations) in a time window $[0, T]$, we can determine its Fourier transform by $\tilde{Z}=\int_{0}^{T} d t Z(t) e^{2 \pi i f t}$; its power spectrum is then defined as the scaled variance of the Fourier transform

$$
S_{Z Z}(f)=\frac{\left\langle\tilde{Z} \tilde{Z}^{*}\right\rangle}{T}
$$

where the asterisk denotes complex conjugation. Since the conductance dynamics is just a linear filter acting on the total synaptic input, the power spectra of the conductance fluctuation $S_{G G}$ and that of the total input $S_{X X}$ are related by

$$
S_{G G}(f)=\frac{\left(\tau g_{0} / N\right)^{2}}{1+(2 \pi f \tau)^{2}} S_{X X}(f) .
$$

For the latter spectrum, we find for low input rate $r$ the following approximation (see Appendix):

$$
\begin{array}{r}
S_{X X} \approx r N\left\langle A_{i, j}^{2}\right\rangle+2 r^{2} N\left\langle A_{i, j}\right\rangle \times\left[\frac{\Delta \tau_{F}}{1+\left(2 \pi f \tau_{F}\right)^{2}}-\frac{F_{0}{ }^{2} \tau_{D}}{1+\left(2 \pi f \tau_{D}\right)^{2}}\right. \\
\left.-\frac{\Delta F_{0} \tilde{\tau}}{1+(2 \pi f \tilde{\tau})^{2}}\right],
\end{array}
$$

where $A_{i, j}=F_{i, j} D_{i, j}$ and $\tilde{\tau}=\left(\tau_{F}^{-1}+\tau_{D}^{-1}\right)^{-1}$. An approximation for the second moment of the synaptic amplitude needed in this formula is sketched in Appendix.

Rate-modulated input and quantifying information transmission. We modulate the rate of the Poisson process that drives the synaptic input in two different ways: first, by a sinusoidal signal $R_{\text {sin }}(t)$ with frequency $f_{s}$, and then by a band-limited Gaussian signal $R_{\text {ran }}(t)$ (cutoff frequency 50 $\mathrm{Hz}$ ). For sinusoidal signals, we use the signal-to-noise ratio (SNR) to quantify the response (McNamara and Wiesenfeld, 1989; Gammaitoni et al., 1998). For weak periodic driving, the power spectrum contains peaks at the driving frequency (inversely proportional to the frequency bin) and the negative driving frequency, on top of a continuous background spectrum (noise floor $S_{\text {background }}$ ). For a finite simulation time $T_{\text {sim }}=$ $\Delta_{f}^{-1}$, the spectrogram of the conductance $S_{G G}\left(f=i_{f} \times \Delta_{f}\right)$ reads

$$
S_{G G}=S_{\text {background }}\left(i_{f} \times \Delta_{f}\right)+\frac{\varepsilon^{2}}{4 \Delta_{f}^{2}} g_{1}^{2}\left(\delta_{i f, i f \mathrm{~s}}+\delta_{i f,-i f \mathrm{~s}}\right),
$$

where $\delta_{i, j}$ is the Kronecker function and $g_{1}$ is the amplitude of the timedependent mean value of the conductance. The signal-to-noise ratio is calculated by dividing the peak at the driving frequency by the continuous background spectrum which we obtain from an interpolation over the frequency bins surrounding $i_{f_{s}}$.

For the Gaussian signals, we measured the cross-spectra between the rate-modulated input and the outputs (conductance or output spike train of the LIF model) and calculated from these the coherence function (Rieke et al., 1996; Gabbiani and Koch, 1998):

$$
C_{Z R}=\frac{\left|S_{Z R}(f)\right|^{2}}{S_{Z Z}(f) S_{R R}(f)}
$$

where $S_{Z R}(f)$ is the cross-spectrum and $S_{Z Z}(f)$ and $S_{R R}(f)$ are the respective power spectra of the output $Z$ and the input $R_{\text {ran }}(t)$.

For the coherence data we simulated 5000 realizations over a time window of $\sim 10^{6}$ time steps of $\Delta t=4 \times 10^{-4} \mathrm{~s}$; cross-spectra and power spectra were averaged over 20 frequency bins (we ensured that this did not smooth out any true frequency dependencies) to obtain smooth estimates of the coherence function.

Designing static synapses for comparison to dynamic synapses. We want to compare the statistics of a postsynaptic spike train generated by an LIF with $F D$ dynamics (i.e., with dynamic synapses) to the same kind of statistics for static synapses, i.e., synapses without $F D$ dynamics. A first approach might be to use a constant synaptic amplitude equal to the mean amplitude of the FD synapse in the conductance dynamics (i.e., $g_{0}$ $=\langle F D\rangle$ ). It turns out that the resulting variability of the conductance in this case is much lower (because the amplitude of the presynaptic input does not vary); thus, the LIF output and consequently the LIF's coherence with the rate modulation is drastically lower as well. A better approach is to set up the synaptic conductance dynamics such that the mean and variance of the conductance are approximately the same for static and dynamic synapses. To this end we proceed as follows. In a first simulation, mean and variance of the conductance $\langle G\rangle$ and $\left\langle\Delta G^{2}\right\rangle$ are 
A static synapse

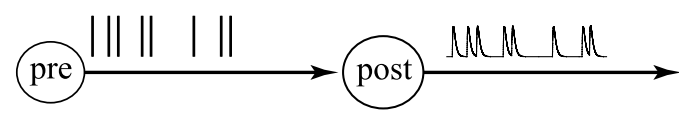

dynamic synapse

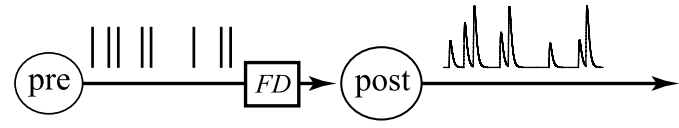

C

presynaptic population

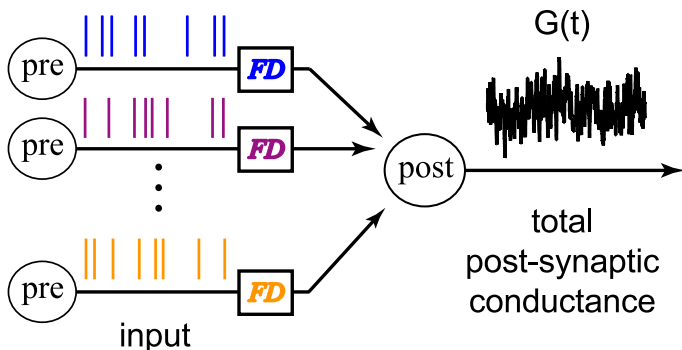

spike trains
B

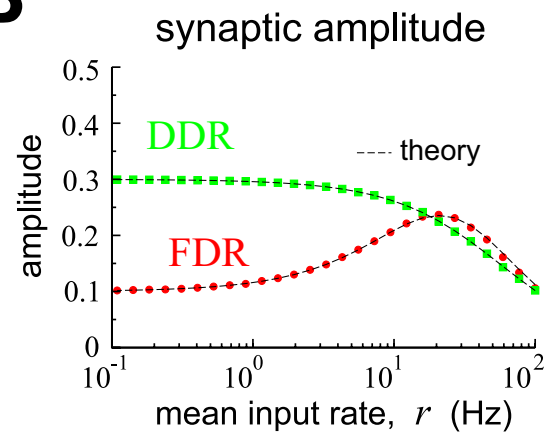

D
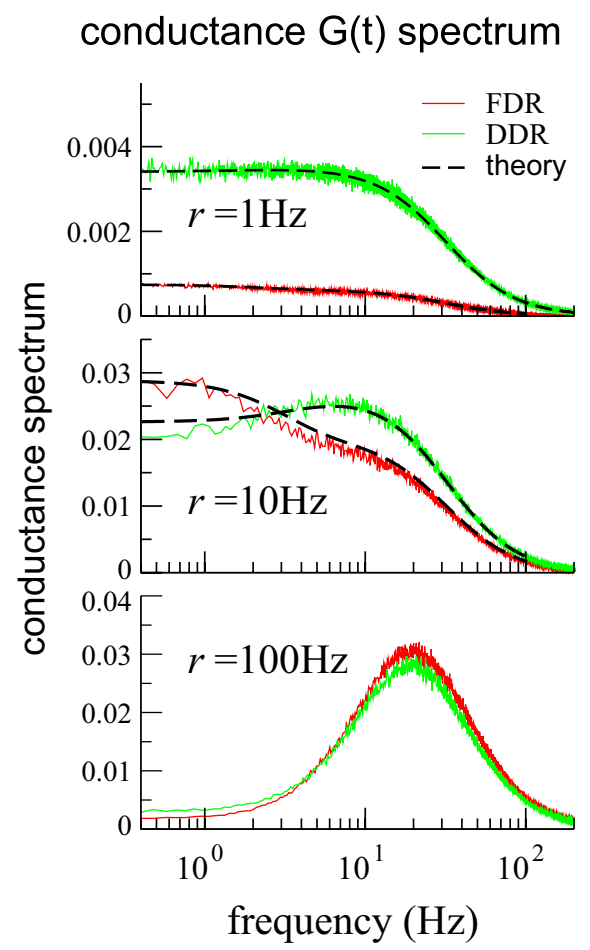

Figure 1. Properties of synaptic dynamics for constant mean input rates. $\boldsymbol{A}$, Schematic illustration of the influence of a single presynaptic spike train (pre) on the synaptic response in a postsynaptic neuron (post), under two different synaptic conditions. Top, Static synapse, with no short-term plasticity and constant synaptic response amplitude; Bottom, Dynamic synapse, with facilitation-depression FD-dependent plasticity and varying synaptic amplitude. $\boldsymbol{B}$, Mean of the synaptic amplitude (dynamic synapse) versus mean input rate $r$ of the Poissonian synaptic inputs. Parameters are $\Delta=0.05, F_{0}=0.3$ for the depression-dominated regime (DDR, green) and $\Delta=0.23, F_{0}=0.1$ for the facilitation-dominated regime (FDR, red); $\tau_{F}=79 \mathrm{~ms}, \tau_{D}=83 \mathrm{~ms}$ for both regimes. Each point for the simulations (symbols) was calculated over $10^{5}$ input spikes. The theory (dashed line) is outlined in Appendix. $\boldsymbol{C}$, Schematic illustration of the influence of a population of presynaptic spike trains (pre) on the net synaptic conductance $G(t)$ in the postsynaptic neuron (post), under FD-dependent plasticity (different colors emphasize the independence of the individual inputs; every synaptic input fires independently with mean rate $r$ ). $D$, Frequency content (power spectra) of the fluctuations in net synaptic conductance $G(t)$ due to $N=100$ independent synapses, for the two different parameter sets used in Figure $1 B$ (DDR, green; FDR, red). Mean input rate $r$ was $1 \mathrm{~Hz}$ (top), $10 \mathrm{~Hz}$ (mid panel), and $100 \mathrm{~Hz}$ (bottom); theory (dashed lines) is compared with simulation results as indicated. Since the theory assumes small input rate, it fails at $100 \mathrm{~Hz}$ (and is therefore not shown; bottom). See Materials and Methods for details.

measured for a population of dynamic synapses. For the static-synapse setup, we then choose the following dynamics for $g_{s}(t)$ :

$$
\dot{g}_{s}=-\frac{g_{s}-\delta_{1}}{\tau}+\delta_{2} \sum_{i, j} \delta\left(t-t_{i, j}\right) .
$$

The task solved in the following is to determine the parameters $\delta_{1}$ and $\delta_{2}$ such that

$$
\left\langle g_{\mathrm{s}}\right\rangle=\langle G\rangle,\left\langle\Delta g_{\mathrm{s}}^{2}\right\rangle=\left\langle\Delta G^{2}\right\rangle .
$$

The first condition together with a steady-state average of Equation 10 [the left-hand side (lhs) vanishes] yields

$$
\delta_{1}=\langle G\rangle-\delta_{2} \tau N r
$$

(here we also assumed that any rectification of the rate modulation can be neglected and hence the mean firing rate over $N$ synapses is just the $N$-fold baseline rate $r$ ). To obtain a second relation, we express the variance of $g_{\mathrm{s}}$ by the integral over the power spectrum

$$
\left\langle\Delta g_{s}^{2}\right\rangle=\int_{-\infty}^{\infty} d f S_{g_{s} g_{s}}
$$

We can easily determine the conductance spectrum $S_{g_{s} g_{s}}$ from the spectrum of the summed constant-amplitude input spike trains $\xi(t)=$ $\Sigma_{j} \xi_{j}(t)$, denoted by $S_{\xi \xi}$, via Fourier transforming Equation 10:

$$
S_{g_{s} g_{s}}=\frac{\left(\delta_{2} \tau\right)^{2} S_{\xi \xi}}{1+(2 \pi f \tau)^{2}}
$$


The spectrum of the summed rate-modulated Poisson process reads [see related calculations by Gabbiani and Koch (1998)]

$$
S_{\xi \xi}=N r+(\varepsilon N r)^{2}\left\langle\tilde{s} \tilde{s}^{\star}\right\rangle=N r+(\varepsilon N r)^{2} \Theta\left(F_{c}-f\right) \Theta\left(F_{c}+f\right) .
$$

Here the product of the Heaviside function $\Theta(\cdot)$ implements the bandpass limitation to frequencies between the cutoffs $-F_{c}$ and $F_{c}$. Inserting the full formula for the spectrum $S_{g_{g} g_{\mathrm{s}}}$ into Equation 13, performing the integral, setting the resulting variance equal to that of the dynamic variance, and solving finally for $\delta_{2}$ yields

$$
\delta_{2}=\left(\frac{2\left\langle\Delta G^{2}\right\rangle \pi / \tau}{2(N \varepsilon r)^{2} \operatorname{atan}\left(2 \pi F_{c}\right)+N r \pi}\right)^{1 / 2} .
$$

Using Equations 16 and 12, $\delta_{1}$ and $\delta_{2}$ can be determined. We note that in all our simulations the static and the dynamic synapses showed very similar mean and variance for the conductance $(<2 \%$ difference); higher-order statistics as well as the tail (e.g., the stationary probability density at high $G$ values) differed slightly.

\section{Results}

\section{Modeling short-term facilitation and depression}

The short-term dynamics of a wide range of synapses are well described by a combination of facilitation and depression, the so-called FD formalism (Abbott et al., 1997; Tsodyks and Markram, 1997; Dittman et al., 2000; Dayan and Abbott, 2001; Lewis and Maler, 2002; Abbott and Regehr, 2004). In FD models, $F$ describes a facilitation process that tends to increase synaptic strength, and $D$ a depression process that tends to decrease synaptic strength, with the net synaptic strength given by the product $F D$. The dynamics of $F$ and $D$ arise from how they change after a presynaptic input spike (Dayan and Abbott, 2001). At the time of each presynaptic spike, $F$ is incremented by a fixed amount $\Delta$ and then decays exponentially toward a baseline value of $F_{0}$ with a time constant $\tau_{F}$. Thus, $F$ can be directly related to the transient increase in calcium concentration due to an action potential in the presynaptic terminal and the associated change in transmitter release probability (Zucker and Regehr, 2002); $F_{0}$ is then the baseline probability of release. Similarly, at each presynaptic spike, $D$ is multiplied by $(1-F)$, then decays back toward 1 with a time constant $\tau_{D}$. The variable $D$ reflects presynaptic $\mathrm{Ca}^{2+}$ channel inactivation (Mochida et al., 2008) and the amount of available transmitter, which is depleted by a fraction $(1-F)$ after each presynaptic spike (Zucker and Regehr, 2002). While the relation to biophysical quantities is not precise, it is important to realize that such models are able to capture a wide range of the shortterm synaptic dynamics observed experimentally (Abbott and Regehr, 2004; Morrison et al., 2008).

In general, as $F_{0}$ increases from 0 to 1 , a synapse changes from being facilitation dominated to depression dominated. Typically, synapses in different neural pathways are dominated by either facilitation or depression, but in some cases can transition between the two regimes over longer timescales (Markram and Tsodyks, 1996; Abbott and Regehr, 2004; Lewis and Maler, 2004). In principle, multiple $F$ and $D$ processes can be used to describe a particular synapse, but we consider here just one of each. As is the convention, we use FD to scale an excitatory synaptic conductance, $G$, having a time constant $\tau$ (see Materials and Methods). A population of these synaptic conductances $(N=100)$, each with independent $F D$ dynamics and independent Poissonian input spike trains, provides the input to an LIF model neuron. To investigate the specific effects of synaptic dynamics without the additional nonlinearities associated with action potential generation, we first consider the condition when spiking is blocked, and then subsequently we describe the effects on output spike trains.

It is important to note that our approach (1) is directly related to many previous studies of synaptic dynamics (Abbott et al., 1997; Tsodyks and Markram, 1997; Dayan and Abbott, 2001; Abbott and Regehr, 2004, and references therein), (2) allows us to consider a range of relative strengths of facilitation and depression in a synaptic pathway, and specifically focuses on two distinct regimes: facilitation-dominated or depression-dominated, and (3) does not address the issues of a single synaptic pathway dynamically switching between facilitation and depression, nor the stochastic nature of synaptic transmission.

\section{Dynamics of synaptic amplitudes with facilitation and depression}

Our analysis focuses first on the mean synaptic amplitude (synaptic gain) as a function of the mean rate $r$ of a single presynaptic input (Fig. 1A,B). Previous studies have shown this relationship to take two qualitatively different forms (Abbott and Regehr, 2004), which we illustrate in Figure $1 B$ with two different parameter sets. The first (red circles) is taken from a experimentally based model described previously (Lewis and Maler, 2004). Here, the amplitude grows with increasing input rate, reaches a maximum at $\sim 20 \mathrm{~Hz}$, and then drops to 0 for high rates. Up to an input rate of $20 \mathrm{~Hz}$, facilitation dominates in the sense that increases in input rate lead to a larger mean synaptic amplitude. We refer to the parameter range of $\left(F_{0}, \Delta\right)$, in which the mean amplitude grows with input rate $r$, as the facilitation-dominated regime or FDR. Strictly speaking, this will of course depend on $r$, but here the definition applies for low to moderate rates. A second parameter set (green squares) results in a monotonic decrease in mean synaptic amplitude with input rate $r$. In this case, depression dominates for the entire range of input rates. We refer to this parameter range as the depression-dominated regime or DDR. Such dynamics in the synaptic amplitudes have led to the hypothesis that facilitation acts as a high-pass filter, and depression acts as a low-pass filter (for review, see Abbott and Regehr, 2004). In this context, high-pass and low-pass refer to the range of input rates for which the mean synaptic amplitude (or gain) is large relative to that for other rates. Later, we will also consider high-pass and low-pass in the context of the frequency at which a baseline Poisson rate is modulated.

\section{Facilitation and depression differentially influence fluctuations in synaptic conductance}

We now consider the effects of a population of synaptic inputs on fluctuations in membrane conductance (Fig. 1C); this is analogous to recording synaptic current in a voltage-clamp experiment. (Note: the context here involves spontaneous activity due to Poisson inputs with constant mean rate; encoding a ratemodulated signal is considered later.) While the synaptic amplitude is a conventional measure of synaptic efficacy and can indicate whether facilitation or depression dominates at a synapse (as illustrated in the previous section), it does not reveal anything about the temporal fluctuations introduced by STP (FD dynamics). Likewise, it does not reveal how these fluctuations shape the noise background at different frequencies, which is relevant for signal detection and transmission. Instead, to investigate these features we use the power spectrum. The power spectrum describes any time-varying signal (membrane current, spike train, etc.) in terms of the relative strength of different frequency components; note that we will reserve the use of the term frequency for this context, and will use rate exclusively to refer to the number of input or output spike events per unit time. 
A presynaptic population

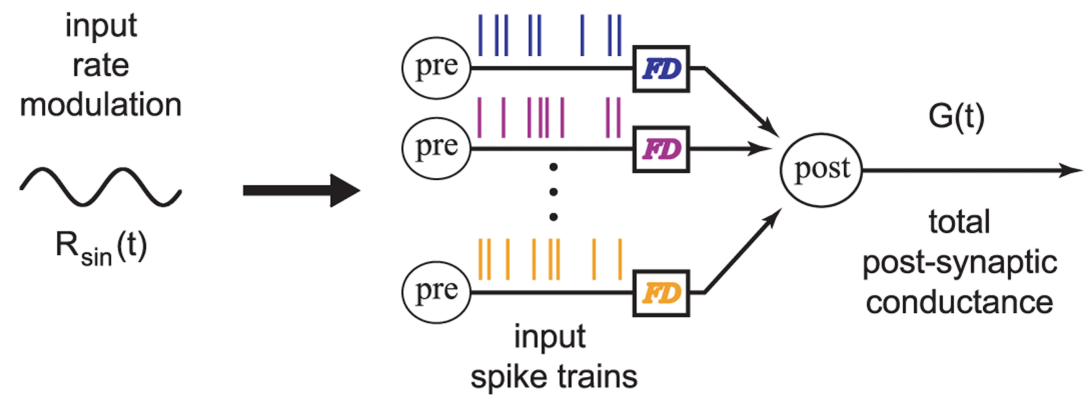

B

$f_{s}=0.5 \mathrm{~Hz}$
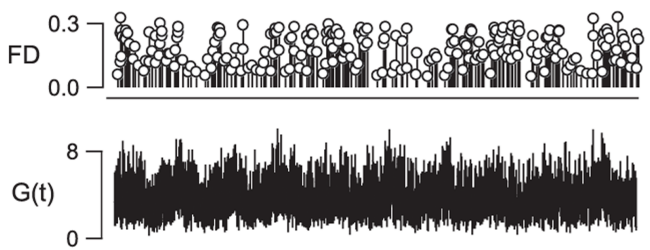

$\mathrm{R}_{\sin }(\mathrm{t})$

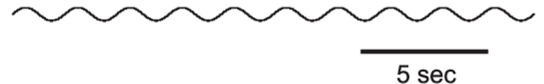

C

FDR

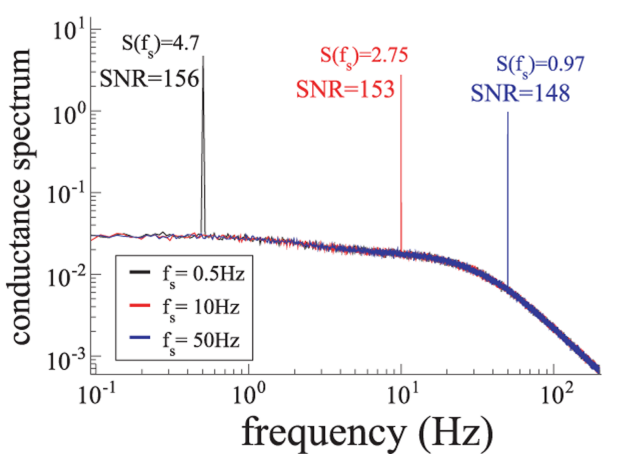

$f_{s}=50 H z$
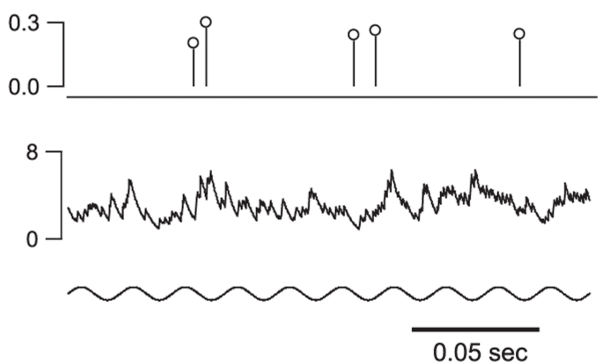

DDR

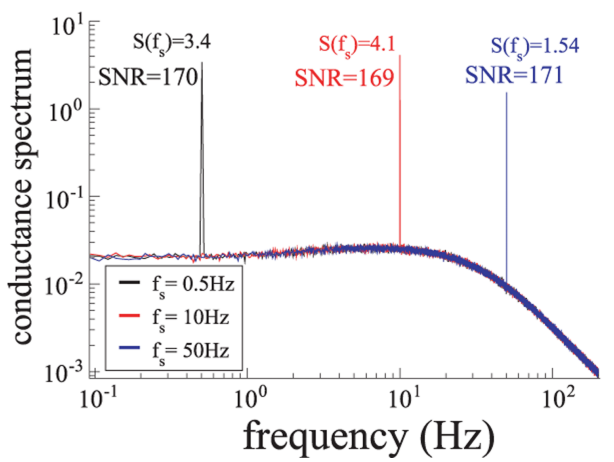

Figure 2. Sinusoidal rate-modulated input signal and the synaptic conductance. $A, A$ schematic illustrating the sinusoidal rate modulation $R_{\text {sin }}(t)$ of a population $(N=100)$ of independent presynaptic inputs onto a postsynaptic neuron. Each input spike train is independent and Poissonian with a mean rate $r$ that is modulated by the signal $R_{\text {sin }}(t)$ as $r\left[1+\varepsilon R_{\text {sin }}(t)\right]$, where $R_{\text {sin }}(t)$ is a unit-amplitude sine wave with frequency $f_{s}$. We apply the same rate modulation to the input spike trains of all synapses, although each is independent. The output in this scenario is the total postsynaptic conductance, $G(t)$. $\boldsymbol{B}$, Representative time series of the amplitude of one FD-modulated synaptic input (top) and the total postsynaptic conductance $G(t)$ (bottom; in $n S$ ), for two values of sinusoidal rate modulation, $f_{\mathrm{s}}=0.5 \mathrm{~Hz}$ (left) and $f_{\mathrm{s}}=50 \mathrm{~Hz}$ (left) with $r=10 \mathrm{~Hz}$ and $\varepsilon=0.1$. C, Frequency content (power spectra) of the fluctuations in net synaptic conductance $G(t)$ for three different forcing frequencies ( $f_{\mathrm{s}}=0.5 \mathrm{~Hz}$, black; $f_{\mathrm{s}}=10 \mathrm{~Hz}$, red; $f_{\mathrm{s}}=50 \mathrm{~Hz}$, blue), using FDR parameters (left) and DDR parameters (right) as in Figure 1. Also indicated is the power at each forcing frequency, $S\left(f_{s}\right)$, and the corresponding signal-to-noise ratio, SNR, as defined in Materials and Methods.

From the power spectrum of the total membrane conductance $G(t)$, we see that the main effect of STP at low and moderate input rates (e.g., mean rate, $r=10 \mathrm{~Hz}$ ) is different for the FDR and DDR (Fig. 1D, middle). In the FDR, conductance fluctuations (spectral power) in the low-frequency ranges are large relative to those in the DDR. Further, in the FDR, spectral power decreases with increasing frequency, whereas in the DDR, spectral power first increases then decreases, with a spectral peak near $7-10 \mathrm{~Hz}$. Note that for low to moderate frequencies, the effect of facilitation and depression is the opposite of that observed for the mean synaptic amplitude (Fig. $1 B$ ): here, facilitation leads to low-pass filtering, while depression implements a high-pass filter. At very high input rates $(r=100 \mathrm{~Hz})$ how- ever, depression dominates for both parameter sets, resulting in low power in the low-frequency ranges for both conditions (Fig. $1 D$, bottom). In all cases, the loss of power in the high-frequency range $(>30 \mathrm{~Hz})$ is due to depression in combination with the conductance dynamics, which effectively low-pass filter the input spike train (see Materials and Methods).

\section{Sinusoidal modulation of input rate and the postsynaptic conductance}

We now consider a simple example in which a sinusoidal signal is transmitted across the synapse. We assume the signal is initially encoded by a sinusoidal modulation of the presynaptic spike rate; 


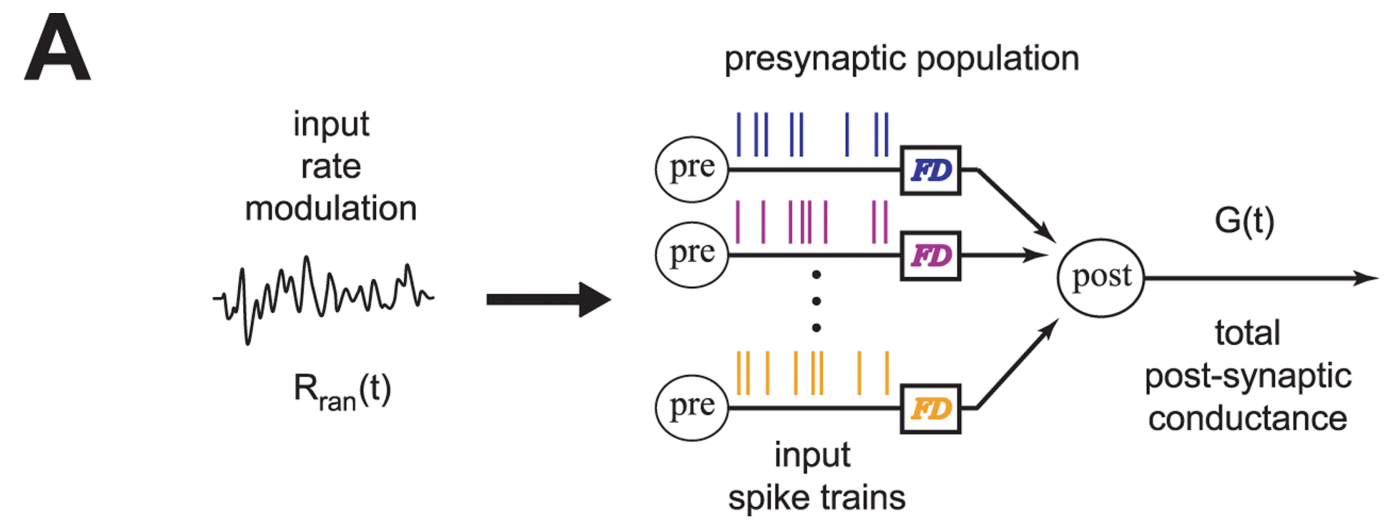

B

G-R cross-spectra

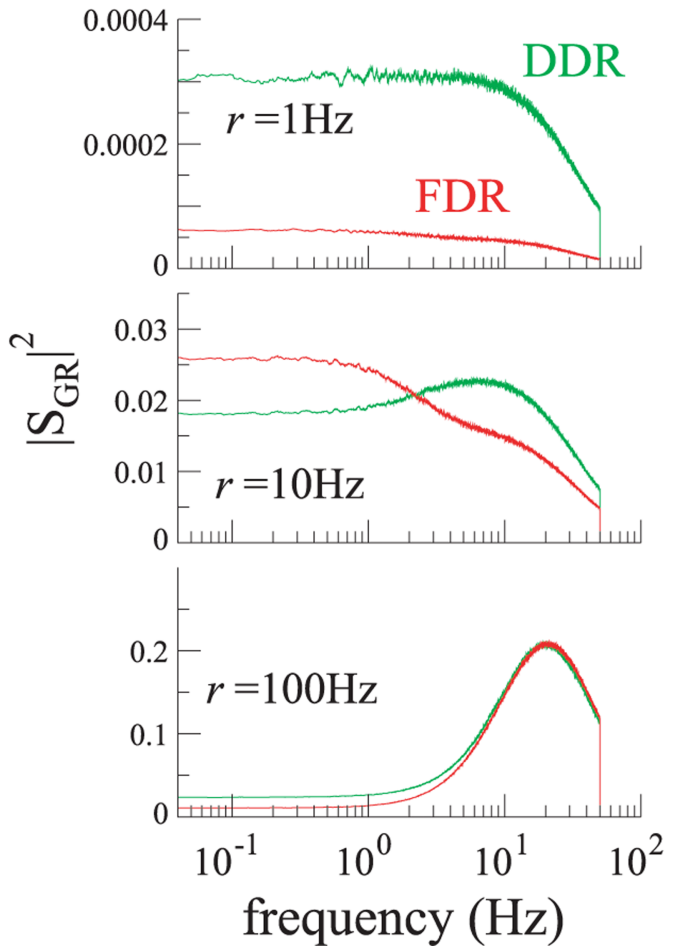

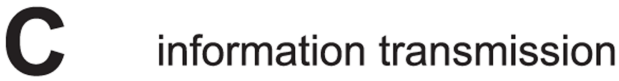
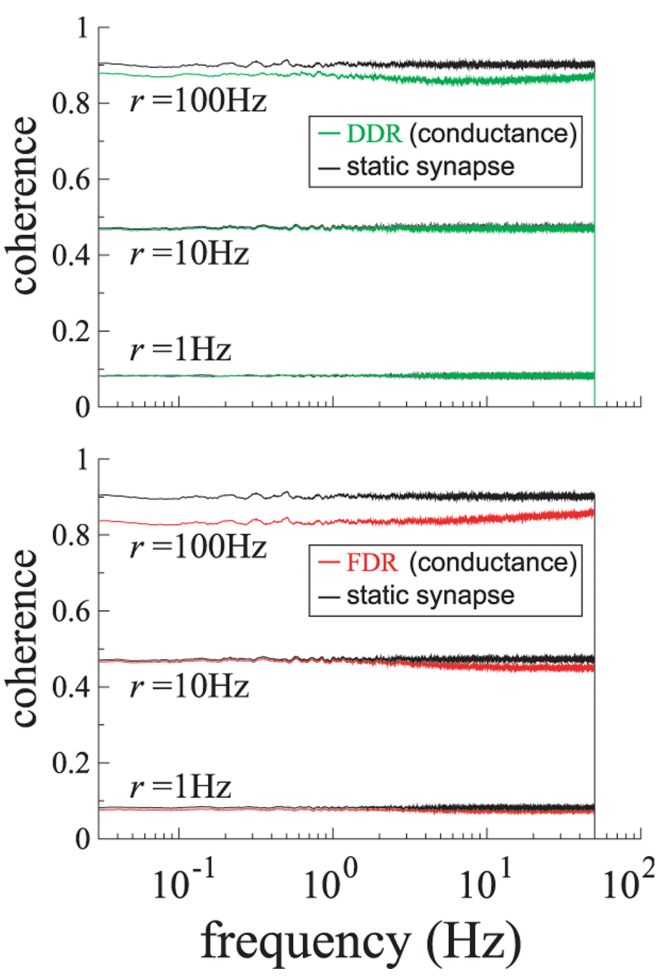

Figure 3. Information transmission of a rate-modulated signal via the synaptic conductance. $A$, A schematic illustrating the rate modulation $R_{\text {ran }}(t)$ of a population $(N=100)$ of independent presynaptic inputs onto a postsynaptic neuron. Each input spike train is independent and Poissonian with a mean rate $r$ that is modulated by the signal $R_{\text {ran }}(t)$ as $r\left[1+\varepsilon R_{\text {ran }}(t)\right]$, where $R_{\text {ran }}(t)$ is band-limited $\left[0-50 \mathrm{~Hz}\right.$ ] Gaussian white noise and $\varepsilon=0.03$ [corresponding to a $30 \%$ change in the input rate for one SD of $R_{\text {ran }}(t)$ ]. The same rate modulation is applied to the input spike trains of all synapses, although each is independent. $\boldsymbol{B}$, The squared cross-spectra of the rate modulation $R_{\text {ran }}(t)$ with the total synaptic conductance for the DDR (green) and the FDR (red); parameter values are as in Figure 1 with baseline (mean) input rate $r$ as indicated. Note the great similarity to the power spectra shown in Figure 1D.C, Information transmission between band-limited rate modulation $R_{\text {ran }}(t)$ and synaptic conductance $G(t)$ as quantified by the coherence function. As in $B$, the two parameter sets from Figure $1 B$ are used for the DDR (green) and FDR (red). Also shown is the coherence for synaptic input with constant amplitude (static synapses, in black). Baseline rates are $r=1 \mathrm{~Hz}, 10 \mathrm{~Hz}$, and $100 \mathrm{~Hz}$ as indicated.

this rate modulation is referred to as $R_{\text {sin }}(t)$, which denotes a pure sine wave of amplitude 1 and frequency $f_{s}$. In other words, the Poisson firing rate of the population of 100 synaptic inputs is modulated by $R_{\text {sin }}(t)$, as $r\left[1+\varepsilon R_{\text {sin }}(t)\right]$, where $r$ is the mean input rate and $\varepsilon$ is the amplitude of the rate modulation. This scenario is outlined schematically in Figure $2 A$. Figure $2 B$ shows the $F D$ dynamics (FDR) for a single input (synaptic amplitudes) and the total postsynaptic conductance $G(t)$ for two different sinusoidal rate modulations $\left(f_{\mathrm{s}}=0.5\right.$ and $50 \mathrm{~Hz}$; mean rate $r=10 \mathrm{~Hz} ; \varepsilon=$ 0.1 ). Because of the large fluctuations involved, it is difficult to visually assess the transmission of the sinusoidal signal. In this context, signal transmission can be quantified by the conductance power spectra and the signal-to-noise ratio, SNR (Fig. 2C, left) (see also Materials and Methods). Note that the SNR varies very little for the three sinusoidal frequencies tested $(\mathrm{SNR}=156$, 153 , and 148 for $f_{\mathrm{s}}=0.5,10$, and $50 \mathrm{~Hz}$, respectively; FDR). This is also true in the DDR (Fig. $2 C$, right), although comparatively, the SNR is slightly greater $\left(\mathrm{SNR}=170,169,171\right.$ for $f_{\mathrm{s}}=0.5,10$, and $50 \mathrm{~Hz}$, respectively; DDR). As we will show in the following sections, the fact that these different rate-modulated signals result in a relatively constant SNR (for both FDR and DDR) has important implications for synaptic information transmission. 
A

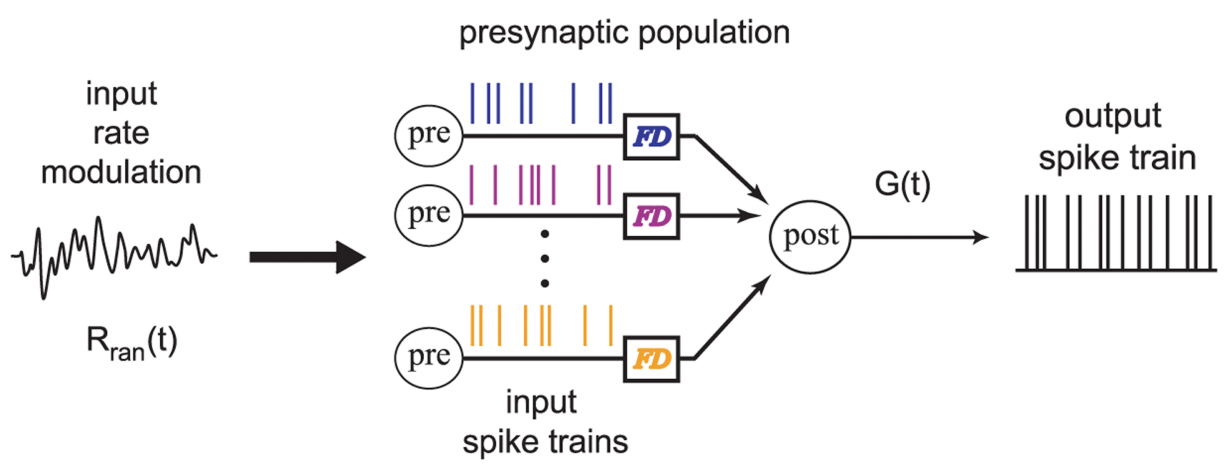

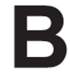

DDR

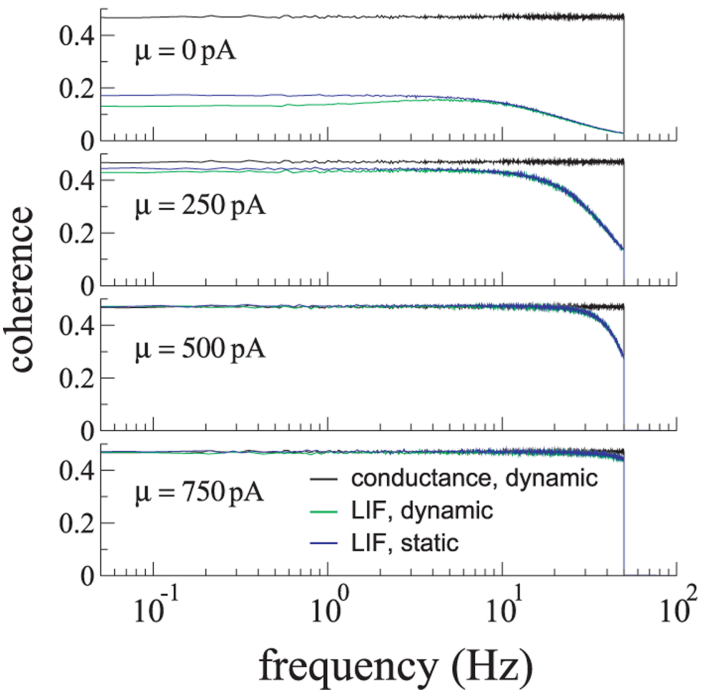

C

FDR

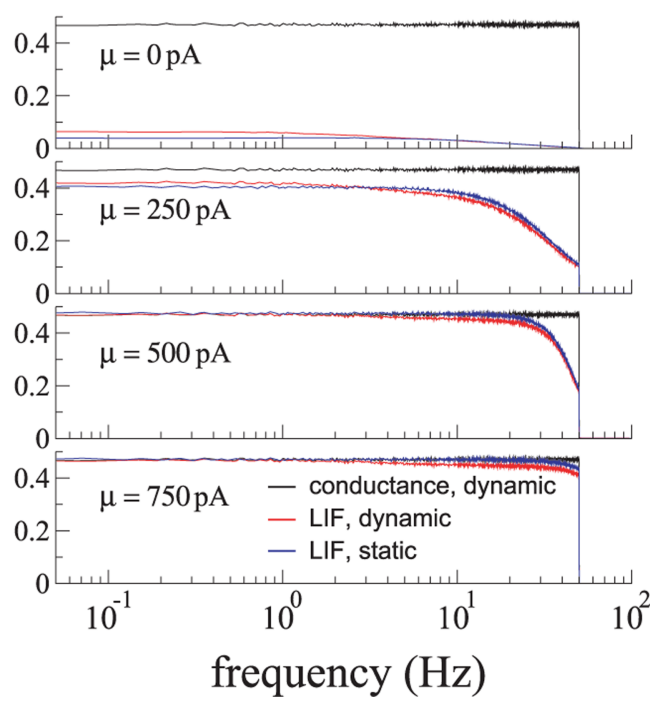

Figure 4. Information transmission of a rate-modulated signal via the output spike train. $\boldsymbol{A}$, The extended scheme that includes an LIF model neuron generating an output spike train. $\boldsymbol{B}$, The coherence between the rate modulation of the input spike trains $R_{\text {ran }}(t)$ and the output spike train of the LIF, for different values of a constant bias current $\mu$ applied to the LIF neuron (all for the DDR parameter set, $r=10 \mathrm{~Hz}$ ). At zero bias current $\mu$ (top), the coherence for the FD-modulated synapse (green) is lower at low frequencies than for static synapses (blue) and much lower than for the conductance (black; same as Fig. 3C). With increasing value of the bias current ( $\mu=250,500,750 \mathrm{pA}$ ) and thus of the output firing rate, the coherence increases and becomes flat, similar to the conductance coherence; the difference between static and dynamic (FD-modulated) synapses consequently vanishes. $\boldsymbol{C}$, Same as in $\boldsymbol{B}$ except for the FDR parameter set. At zero bias current, there is higher coherence at low frequency for the dynamic (FD-modulated) synapses than for the static synapses. Again, with increasing $\mu$, the differences vanish; the coherence increases and approaches the coherence of the conductance, thus indicating a linear transfer of information.

\section{Information transmission of a rate-modulated signal to the postsynaptic conductance}

While sinusoidal rate modulations can be instructive, to quantify information transmission in a general sense, we use a more complex signal to drive the rate modulation of the presynaptic input spike trains. Specifically, this signal is taken as a band-limited [0, $50 \mathrm{~Hz}$ ] Gaussian random signal $R_{\text {ran }}(t)$. The Poisson firing rate of the population of 100 synaptic inputs is then modulated by $R_{\text {ran }}(t)$, as $r\left[1+\varepsilon R_{\text {ran }}(t)\right]$. To quantify information transmission between the input signal $R_{\text {ran }}(t)$ and a particular output $Z(t)$, we use the spectral coherence, $C_{Z R}$ (Eq. 9) (Rieke et al., 1996; Gabbiani and Koch, 1998). The coherence $C_{Z R}(f)$ is a measure that ranges, at each frequency, between 0 (in which case the output is not linearly related to the input at that frequency, due to noise or nonlinearity) and 1 (in which case there is perfect linear transmission of the input stimulus at that frequency). For weak stimuli, a lower bound on the mutual information rate is directly related to the spectral coherence (Rieke et al., 1996). As in the previous sections, we consider the total synaptic conductance
$G(t)$ as our output measure (Fig. 3A), and thus the cross-spectra $S_{G R}(f)$ of the rate modulation with the synaptic conductance. Note that the cross-spectrum is directly proportional to the frequency-dependent gain of the system (also commonly referred to as the transfer function). Surprisingly, for both FDR and DDR, the shape of the squared cross-spectra (Fig. $3 B$ ) is very similar to that of the power spectra $S_{G G}$ seen for the unmodulated condition (i.e., Poisson inputs with fixed mean rate) (Fig. $1 D$ ). In the FDR, the cross-power decreases with frequency for low and moderate input rates $(r=1$ and $10 \mathrm{~Hz}$ ); in this case, the synaptic dynamics act as a low-pass filter on the rate modulation. In the DDR, power is relatively suppressed in the low-frequency range for moderate to high input rates $(r=10$ and $100 \mathrm{~Hz})$; the synaptic dynamics, in this case, act as a high-pass filter from the cross-spectral point of view. For very low input rate $(r=1 \mathrm{~Hz})$, low-pass behavior is also seen for DDR. We note for reference that a static synapse (constant synaptic amplitude with Poisson input spike train; see Materials and Methods) leads to a flat cross-spectrum which, 


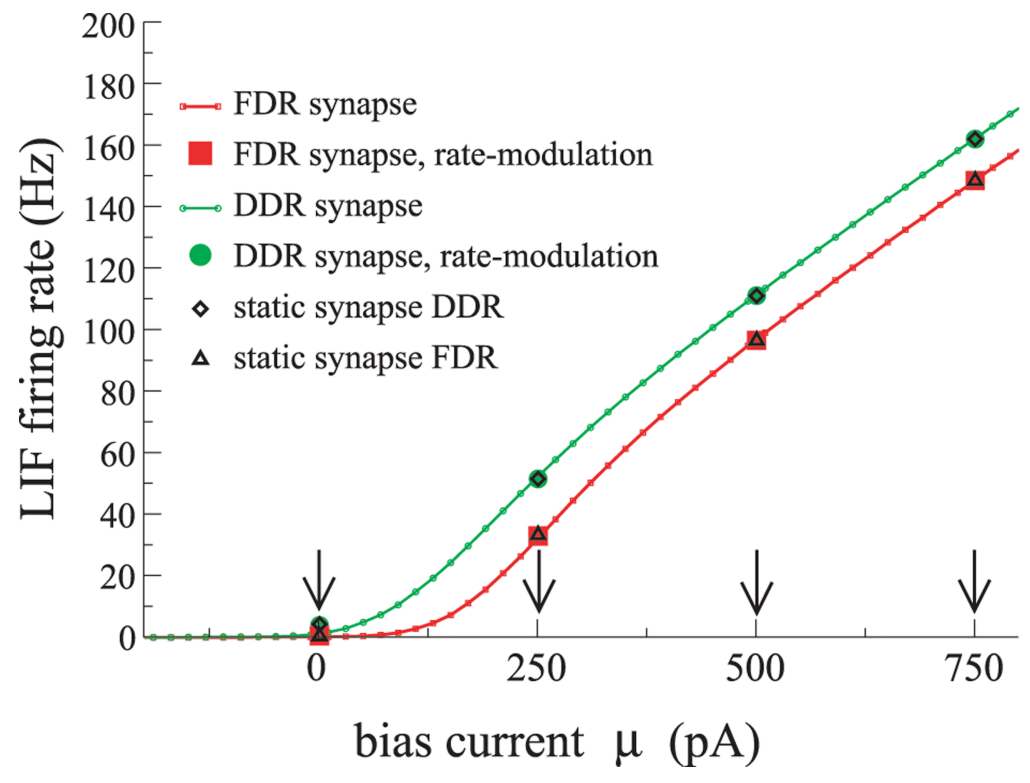

Figure 5. LIF model neuron firing rate under different synaptic input conditions Firing rate versus constant bias current $\mu$ for the different conditions considered in the present analyses; FDR and DDR indicate the particular parameter sets as in previous figures; mean input rate $r=10 \mathrm{~Hz}$. FDR, DDR synapse: stationary input as in Figure 1; FDR, DDR synapse rate modulation: random rate modulation as in Figure 4; FDR, DDR static synapse: static synapse with same mean and variance as FD-modulated synapse for each regime (as in Fig. 4) (see Materials and Methods for additional details). Arrows indicate the values of bias current used in Figure 4.

for a weak rate modulation, can also be derived analytically (Gabbiani and Koch, 1998).

The important consequences of the similarities between Figures $1 D$ and $3 B$ become evident when we consider the coherence between the rate modulation and the conductance, $C_{G R}$. First, overall coherence increases with growing mean input rate $r$ (Fig. $3 C)$. More importantly however, for both FDR and DDR, the coherence is flat with respect to the rate modulation of the input [frequency content of the signal $R_{\mathrm{ran}}(t)$ ]. In other words, the $F D$ dynamics do not result in a frequency-dependent transfer of information. Such a flat, or broadband, coherence coding property is usually expected for a linear system with additive white noise and white input signal. In particular, the static synapse case shown in Figure $3 C$ is a close approximation of this linear case; the coherence is flat because the cross-spectrum and power spectrum are similarly low-pass (data not shown). However, in the present context (a band-limited signal encoded in presynaptic spike trains driving nonlinear synaptic dynamics), this broadband property is unexpected. Indeed, it arises because the power spectra of the membrane conductance fluctuations $G(t)$, with and without the signal $R_{\text {ran }}(t)$, are both similar in shape to the cross-spectra between $R_{\text {ran }}(t)$ and $G(t)$; thus, their ratio in the coherence function cancels out any frequency dependence. In other words, in the FDR/DDR, STP not only results in increases/ decreases in mean synaptic amplitude, but it also acts to low-pass/ high-pass filter the rate-modulated signal. The combination of these two directly related effects results in a frequencyindependent transfer of information to the postsynaptic conductance.

Further, a peak in the power spectra of the conductance fluctuations at frequencies near $10-20 \mathrm{~Hz}$ is seen for the DDR (Fig. $1 D$, middle and bottom), as well as for the FDR (at high rates when depression dominates) (Fig. $1 D$, bottom). Such a peak is also present in the cross-spectra (Fig. $3 B$ ) for the same mean rates, $r$. These peaks, being associated with dominant-depression, sug- gest an increased sensitivity to the highfrequency content of a rate-modulated signal. This is in contrast to the synaptic amplitude-versus-input rate plots (Fig. $1 B)$, which show a peak for the FDR and low-pass behavior for the DDR. These contrasting behaviors (Figs. $1 D, 2 B$ ) are at the root of the previously observed enhanced response of depressing synapses to abrupt changes in the input rate, when firing rates are sufficiently high (Abbott et al., 1997; Abbott and Regehr, 2004).

\section{Information transmission of a rate- modulated signal to the output spike train}

Thus far, our analyses have been limited to information encoded in the synaptic conductance. In the context of information transfer, it is of course critical to consider the membrane potential fluctuations and the spiking dynamics of the postsynaptic neuron. Under conditions in which spiking is blocked (subthreshold membrane potential), the membrane potential behaves similarly to the synaptic conductance (data not shown).

We now consider a spiking LIF neuron being driven by dynamic synaptic inputs (Fig. 4A). This situation is identical to that described for the synaptic conductance (Fig. 3), but with the conductance now driving the membrane potential and an LIF spiking mechanism (see Materials and Methods). In addition, we consider both the DDR (Fig. $4 B$ ) and the FDR (Fig. 4C) compared with static synapses having no STP but causing conductance fluctuations with the same mean and variance as the respective dynamic synapses (see Materials and Methods). To separate out the effects of the LIF itself from the synaptic dynamics arising from STP, we also apply different levels of a constant bias current $(\mu)$. In doing so, we find that the coherence between the input rate modulation and the spike output depends on the firing regime of the LIF. When the bias current is large, causing the neuron to fire at a high rate, the broadband coding observed for the synaptic conductance (Fig. 3) is preserved for both DDR and FDR (and is essentially the same as that for the static synapses). However, for decreasing levels of bias current, such that the mean firing rate of the LIF neuron decreases, the STP-dependent filtering becomes apparent in the low-frequency range (compare with static synapse, $\mu=0 \mathrm{pA}$ ) (Fig. $4 B, C$ ). In addition, the increased nonlinearity of the LIF in this regime $(\mu=0 \mathrm{pA})$ results in a drop in coherence in the high-frequency range for both DDR and FDR. Figure 5 illustrates that the LIF firing rates for the FDR and DDR, both in the unmodulated and rate-modulated cases, are essentially identical to their respective matched static synapses, for a given bias current $\mu$, and thus cannot be the cause of any differences between static and dynamic synapses. However in general, the lower coherence for the FDR is due to a slightly lower firing rate of the LIF in the FDR (Fig. 5).

Thus, we find that information transmission via the output spike train depends on the state of the LIF neuron: broadband coding is preserved when the neuron is well above threshold, but filtering due to both STP and LIF dynamics is apparent when the neuron is near threshold $(\mu=0 \mathrm{pA})$, in the so-called fluctuation- 

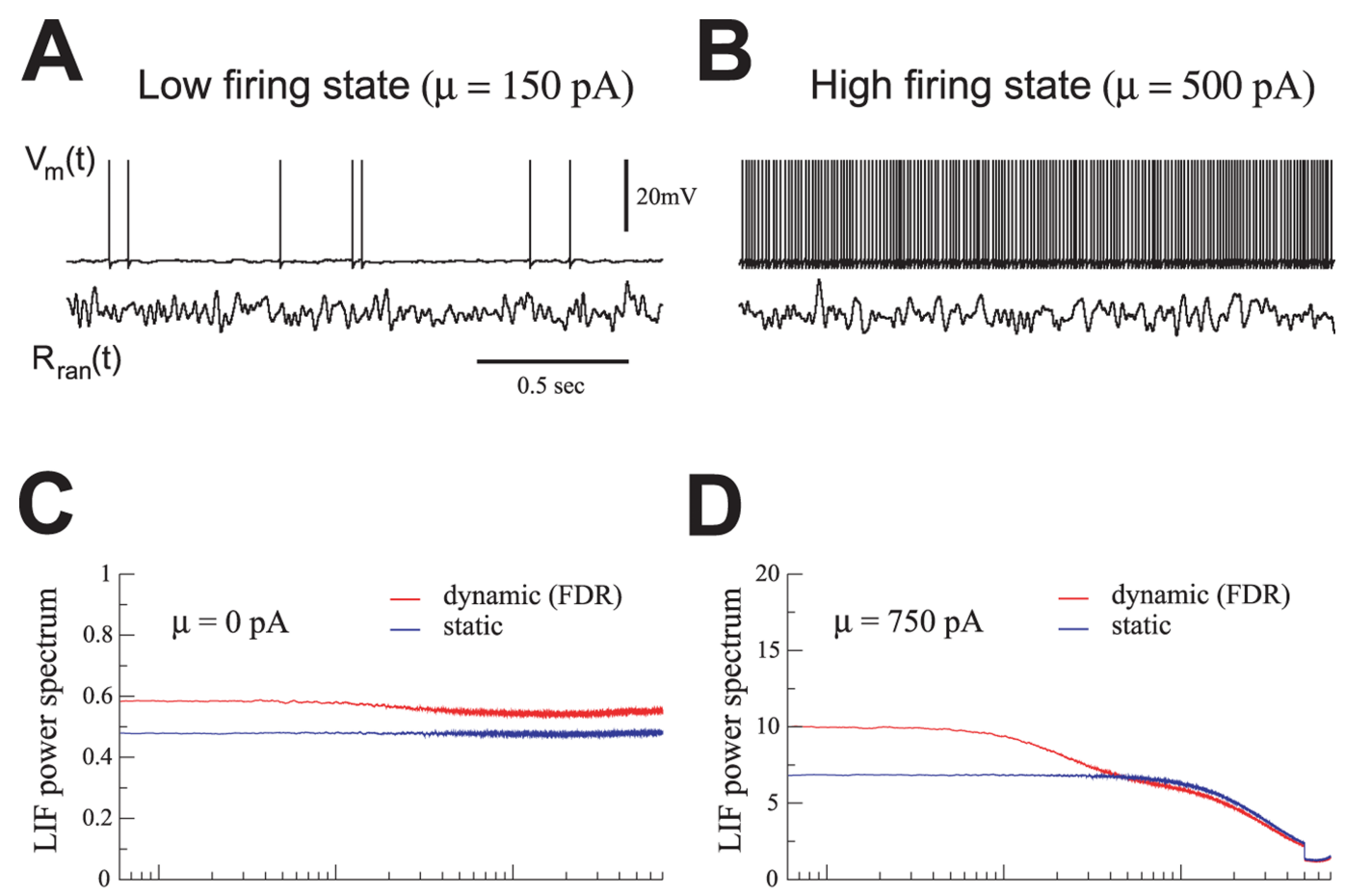

D
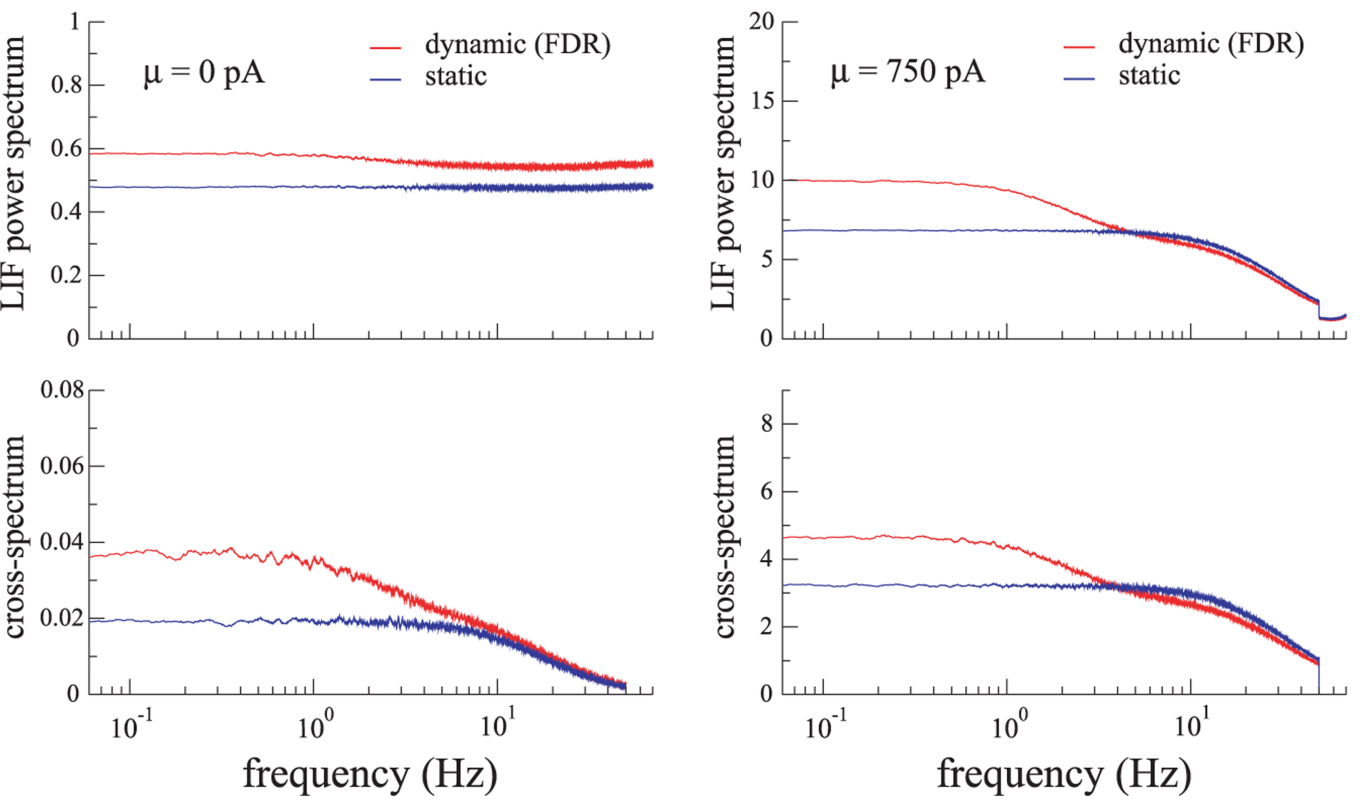

Figure 6. Spectral features of the LIF output spike train in low-and high-firing states. $A, B$, Example traces of LIF output spike trains in a low-firing state $(\mu=150 \mathrm{pA})$ and a high-firing state $(\mu=$ $500 \mathrm{pA}$ ), respectively. Both are responses to the rate-modulated input $R_{\text {ran }}(t)$ with mean input rate $r=10 \mathrm{~Hz}$ and $\varepsilon=0.03$ using the FDR parameter set. As is the convention for LIF model spike trains, spikes are drawn in as vertical lines at the times when the membrane potential reaches threshold. Note that these intermediate values of bias current were chosen for illustrative purposes; $\mu=0 \mathrm{pA}$ and $\mu=750 \mathrm{pA}$ result in firing rates that are too low and too high, respectively, to be easily compared on the same timescale. $C$, The spike train power spectra (top) and the cross-spectra (bottom) between the input rate modulation and the output spike train for a low-firing state $(\mu=0 \mathrm{pA})$. $\boldsymbol{D}$, The spike train power spectra (top) and the cross-spectra (bottom) between the input rate modulation and the output spike train for a high-firing state $(\mu=750 \mathrm{pA})$. In both $\mathbf{C}$ and $\boldsymbol{D}$, the rate modulation $R_{\text {ran }}(t)$ is the same as that used in Figure 4 , with mean input rate $r=10 \mathrm{~Hz}$; the dynamic synapse refers to the facilitation-dominated parameter regime (FDR, in red), with the static synapse shown in blue.

driven regime (where spike threshold is crossed only for sufficiently large fluctuations). In this latter case ( $\mu=0 \mathrm{pA})$, the LIF spike train is dominated by fluctuation-driven firing such that its power spectrum is whitened (i.e., it becomes flatter indicating more Poisson-like firing). We illustrate the significance of this for the FDR in Figure 6 (DDR is similar). Example LIF spike trains are shown for $\mu=150 \mathrm{pA}$ (low-firing state, fluctuation-driven firing) (Fig. 6A) and $\mu=500 \mathrm{pA}$ (high-firing state) (Fig. 6B). Note that we use intermediate values of $\mu$ to make it easier to compare the two spike regimes on the same timescale. The LIF spike train power spectra for low- and high-firing states are shown in Figure 6, $C$ and $D$ (top); it is clear that the spectra are more flat for $\mu=0 \mathrm{pA}$. Figure $6, C$ and $D$ (bottom), shows the cross-spectra between the input, $R_{\mathrm{ran}}(t)$, and the LIF spike trains. Now, recall that coherence is related to the ratio of the crossspectrum and power spectrum (Eq. 9). The consequence of the flat power spectra (Fig. 6C, top) is that the coherence now reflects the filtering properties exhibited by the cross-spectra (Fig. 6C, bottom). On the other hand, when the power spectra reflect the same frequency dependencies as the cross-spectra, the coherence is flat. In a more natural condition, this bias current could arise from populations of independent background, or feedback, inputs (mediated through glutamatergic synapses), and thus would not be constant but would include additional fluctuations and noise. If these inputs had zero mean (similar to the $\mu=0 \mathrm{pA}$ case), the output spike train power spectra would be even more flat, such that the coherence would reveal the same filtering effects shown by the cross-spectra (data not shown). For large mean input levels (similar to $\mu=750 \mathrm{pA}$ ), the main effect of adding noise would be to drastically decrease the overall coherence; for the remaining coherence, a modest effect of the synaptic filter, in terms of suppression or enhancement of coherence at low frequencies, can be observed (data not shown).

Overall, these results suggest that synaptic filtering depends critically on the state of the postsynaptic neuron and thus may be actively controlled by background synaptic inputs. More specifically, when a neuron is tonically firing at a high rate, neural coding will not be influenced by STP, whereas in the low-firing- 


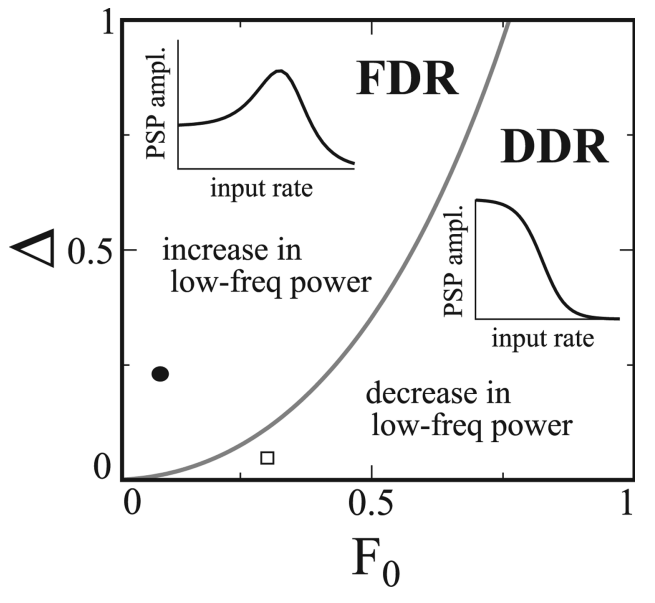

Figure 7. Illustration of parameter space separating DDR and FDR. For the regions in the $\left(F_{0}\right.$ $\Delta$ ) parameter plane below the curved gray line (DDR), the mean synaptic amplitude drops monotonically as a function of the Poisson input rate (inset), and the spectral power of the $F D$-driven synaptic conductance decreases at low frequencies. The curved gray line is defined in Equation 17. In the area above the curve (FDR), the synaptic amplitude displays a maximum versus input rate and the spectral power increases at low frequencies. The parameter sets used in Figure 1 (and in all subsequent analyses) are indicated by the solid circle (FDR) and the open square (DDR); see also Materials and Methods.

rate regimes, more likely associated with spike-timing codes, STP could play an important role in shaping coherence, especially at low frequencies.

\section{Synaptic properties related to the FDR and DDR}

Given that the broadband effect we describe depends on the interaction between synaptic gain (amplitude) (Fig. $1 B$ ) and fluctuations (conductance spectrum) (Fig. 1D), it is important to consider the different parameter regimes more carefully. To do this, we again consider the effects of spontaneous background inputs (as in Fig. 1). Figure 7 shows the $\left(F_{0}, \Delta\right)$ parameter space for both the FDR and DDR (for the related theoretical analyses, see Appendix). By moving along a horizontal line in this plot (at some fixed value of $\Delta$ ), for instance, it is easy to see how changes in $F_{0}$ alone can change a synapse from being facilitationdominated to depression-dominated; in other words, this illustrates how increasing the baseline probability of transmitter release increases the tendency for a synapse to show depression. Most importantly, the diagram illustrates that the distinct behaviors, observed in FDR and DDR, for both the mean synaptic amplitude versus input rate $r$ (existence of a maximum or monotonic decrease) (Fig. $1 B$ ) and the conductance fluctuations (relative increase or decrease in power at low frequencies) are directly related. Indeed, we find the same simple analytical condition on the synaptic parameters for each (gray line in Fig. 7) (see Appendix). The condition for dominating facilitation reads as follows:

$$
\Delta>\frac{F_{0}^{2}\left(1+\tau_{D} / \tau_{F}\right)}{1+\tau_{F} / \tau_{D}-F_{0}} .
$$

The presence of finite timescales $\tau_{D}$ and $\tau_{F}$ is essential for a nontrivial separation of the parameter space: taking one of them to zero we obtain either the trivial condition $\Delta>0\left(\right.$ for $\left.\tau_{D} \rightarrow 0\right)$ or the nonsatisfiable condition $\Delta>\infty$ (for $\tau_{F} \rightarrow 0$ ).

We also observed the broadband coherence effect with various combinations of $\tau_{D}$ and $\tau_{F}$. When these time-constants are $>500$ $\mathrm{ms}$, however, a high-pass effect is found (data not shown). Together, these results show that the relationship between synaptic gain (amplitude) and conductance fluctuations is not due to a delicate balance of parameter values, but rather is expected for a large range of parameters and is thus likely to play a role at many different types of synapses. It should also be noted that a synapse can exhibit facilitation and depression having multiple timescales through distinct overlapping dynamic mechanisms (Zucker and Regehr, 2002; Abbott and Regehr, 2004). The impact of these more complicated scenarios is beyond the scope of the present study and will be addressed in future work.

\section{Discussion}

STP is thought to play various computational roles in neural processing (Abbott and Regehr, 2004). In particular, the notion of synaptic filtering is often used generically to describe how dynamic synapses shape synaptic inputs (Dittman et al., 2000; Lewis and Maler, 2002; Zucker and Regehr, 2002; Abbott and Regehr, 2004; Destexhe and Marder, 2004). The results we present here suggest that this notion, at least under conditions involving the responses to time-varying inputs, should be considered carefully. Indeed, our results suggest that the filtering effects of synaptic dynamics can be controlled by independent inputs via their influence on postsynaptic neuron firing rate.

Given that STP results in synaptic amplitudes that vary with input rates over different timescales, it is reasonable to assume that information transfer through synapses will vary accordingly, with suppression of either high or low frequencies. However, this is not true with respect to the spectral coherence between a ratemodulated input and the STP-modulated postsynaptic membrane conductance. The coherence, which provides a signal-tonoise measure, is flat and independent of frequency. This is because signal transfer (synaptic amplitude) and noise transfer (conductance fluctuations) are increased or decreased in equal proportions at each frequency. Thus, the postsynaptic conductance resulting from a rate-modulated input reflects broadband coding regardless of whether facilitation or depression dominates (Fig. 3).

These results also extend to the dynamics of the subthreshold membrane potential, even though the synaptic conductance is multiplied by the driving force voltage in the Hodgkin-Huxley formalism (see Materials and Methods). However, the threshold dynamics resulting from spiking provide the interesting possibility for the postsynaptic control of synaptic filtering (e.g., through parameters that govern the mean output firing rate). Our results indicate that when the postsynaptic neuron is above threshold and firing autonomously, the broadband coding observed in the membrane conductance and voltage is preserved. However, when the neuron is below threshold and firing is dominated by membrane fluctuations (fluctuation-driven firing, resulting in a flat power spectrum), the synaptic filtering expected from the transfer functions (cross-spectra) in Figure $3 B$ becomes evident.

Previous studies have investigated the effects of STP on input spike trains (Goldman et al., 2002) and information transmission (Fuhrmann et al., 2002). In theory, STP adds a history-dependent amplitude to each spike, so it is expected that in certain cases the correlations of the spike train with the rate modulation will increase. Here, we have shown that this is not equivalent to an increase in information about a time-varying rate signal. Information transmission relies on measuring the signal with respect to a noise background, and thus both signal and noise amplitude are important. From a signal-to-noise perspective, we found that information transfer can be broadband (independent of frequency) in terms of the coherence, even though STP-dependent filtering is present in the transfer function (cross-spectra) (Fig. 
$3 B)$. Interestingly, our results in Figures $1 D$ and $3 B$ reveal that STP in combination with membrane conductance, results in a resonance for both DDR and FDR $(r=100 \mathrm{~Hz})$. In addition, while the coherence exhibits minimal STP-dependent effects, the different features evident in the power spectra and cross-spectra $(r=10 \mathrm{~Hz})$ could provide a method to assess the relative levels of facilitation and depression affecting the synapses onto a neuron of interest.

Short-term plasticity is ubiquitously expressed in neuronal networks. And because our approach is generic and the results do not depend on specific parameter choices, the broadband coding we describe is likely to be operating in a variety of contexts. This has widespread implications for neuronal information processing. First, the fact that the firing state of the postsynaptic neuron can control the influence of STP also presents interesting possibilities for feedback control and the evolution of network organization. Synaptic inputs with identical presynaptic properties could confer differential plasticity solely due to differences in baseline firing rates of postsynaptic neurons. Thus, networks could evolve to extract different information from presynaptic inputs in parallel through postsynaptic neurons with different mean firing rates alone (making distinct control over the presynaptic neurons themselves unnecessary). Second, our results show that any steady-state changes in synaptic gain, due to depression for example (Abbott et al., 1997; Tsodyks and Markram, 1997), will not affect information transmission when the postsynaptic neuron is in the high-firing state. Thus, the prediction follows that pathways where information transmission is important, for example at early stages of sensory processing, will have neurons in a relatively high-firing state, whereas those involved in feature extraction (Gabbiani and Koch, 1998) will comprise neurons in a low-firing state.

One particular example where these issues are relevant is in the electrosensory system of weakly electric fish, the study of which provided the initial motivation for our current analyses (Lewis and Maler, 2002, 2004). The first central nucleus in the electrosensory pathway, the ELL (electrosensory lateral line lobe), receives primary sensory input as well as extensive feedback input. Two classes of ELL projection neurons, the deep (DP) and superficial (SP) pyramidal neurons, are differentiated by their baseline firing rates and the extent to which long-term synaptic plasticity plays a role in their response to feedback inputs (Bastian et al., 2004): DP neurons have high firing rates and are considered nonplastic, whereas SP neurons have low firing rates and very plastic feedback inputs. In light of our present results, DP neurons could reliably encode the rate-modulated feedback inputs across all frequencies (i.e., in a broadband manner), whereas SP neurons could extract particular features of the feedback inputs (Bastian et al., 2004; Oswald et al., 2004).

It is important to realize that our results pertain to timevarying, but statistically stationary modulations of the firing rate of the presynaptic population. Other coding schemes (e.g., a signal encoded in temporal or spatial correlations in firing) or the detection of transient inputs (i.e., abrupt changes in firing rate) may be enhanced by the FD dynamics (Abbott et al., 1997; Lisman, 1997; Abbott and Regehr, 2004). Specifically, STP in excitatory and feedforward inhibitory inputs has been shown to regulate the postsynaptic response to transient inputs (Lewis and Maler, 2002; Klyachko and Stevens, 2006). In such cases, we may see a frequency dependence in information transmission, but appropriate quantification methods are lacking at present. It is also possible that signals much stronger than those we consider here may be able to profit from the information stored in the synaptic amplitudes. Regardless, our results illustrate the potential complexities brought about by synaptic dynamics, and emphasize that appropriate quantitative methods will be essential for evaluating neural coding and transmission of both stationary and transient, nonstationary inputs. More intriguing though, is the possibility that, under some stimulus conditions, the filtering effects of STP can be switched on and off by external inputs simply through their influence on postsynaptic firing rate. The latency of this switch would be short, limited only by the intrinsic neural dynamics. Such a switch is yet another dynamic feature that could be coopted by the neural code.

\section{Appendix}

Here, we derive the statistics of the FD-modulated Poissonian spike train: mean and variance of the amplitude of the synaptic input, as well as its power spectrum. Also derived is the condition Equation 17 for dominating facilitation (i.e., that defines the facilitation-dominated regime, FDR, and the depressiondominated regime, DDR).

\section{Mean synaptic amplitude}

For the dynamics of a single FD model (a single synaptic input $j$ ), we can relate the values of $F_{j}(t)$ and $D_{j}(t)$ right before the $i$ th and $(i+1)$ th input firing times $\left(t_{i, j}, t_{i+1, j}\right)$, denoted by $F_{i, j}, D_{i, j}$ and $F_{i+1, j}, D_{i+1, j}$, respectively. Neglecting the constraint that $F(t)<1$ and omitting the second index $j$ (which indicated the synapse), we obtain the following maps:

$$
\begin{gathered}
F_{i+1}=F_{0}+\left(F_{i}-F_{0}+\Delta\right) e^{-T_{i} / \tau_{F}} \\
D_{i+1}=1+\left(D_{i}-1-F_{i} D_{i}\right) e^{-T_{i} / \tau_{D}},
\end{gathered}
$$

where $T_{i}=\left(t_{i+1}-t_{i}\right)$ denotes the $i$ th interspike interval (ISI). These maps will be a good approximation as long as $\left\langle F_{i}\right\rangle+\Delta<1$ (see also below).

We can use the maps to estimate the mean and variance of the synaptic amplitude $A_{i}=F_{i} D_{i}$ as follows. We can assume that for a renewal spike train (with constant rate) (1) mean values of the form $F_{i+1}^{n} D_{i+1}^{m}$ (with $n, m$ being integer values) will not depend on the index $i$ and thus $\left\langle F_{i+1}^{n} D_{i+1}^{m}\right\rangle=\left\langle F_{i}^{n} D_{i}^{n}\right\rangle$ and (2) the value of a synaptic variable $F_{i}\left(\right.$ or $\left.D_{i}\right)$ is statistically independent of the subsequent interval $T_{i}$. By multiplying powers of the two maps with each other, we obtain a hierarchy of equations for the moment $\left\langle F_{i}^{n} D_{i}^{n}\right\rangle$ containing also averages over the exponentials of the ISI. The latter can be readily calculated for a Poisson process, e.g.,

$$
\left\langle e^{-\frac{T}{\tau_{D, F}}}\right\rangle=\int_{0}^{\infty} d \operatorname{Trexp}\left[-T\left(r+\frac{1}{\tau_{D, F}}\right)\right]=\frac{r}{r+1 / \tau_{D, F}} .
$$

Using this relation and averaging the stochastic maps, we obtain the relations

$$
\begin{gathered}
\left\langle F_{i}\right\rangle=F_{0}+\Delta r \tau_{F}, \\
\left\langle D_{i}\right\rangle=1-r \tau_{D}\left\langle F_{i} D_{i}\right\rangle .
\end{gathered}
$$

From Equation 21 we can conclude that the map Equation 18 is a good approximation as long as 


$$
F_{0}+\Delta\left(1+r \tau_{F}\right)<1
$$

Multiplying the lhs and right-hand side (rhs) of both maps in Equations 18 and 19, followed by averaging, yields the equation

$$
\begin{aligned}
& \left\langle F_{i+1} D_{i+1}\right\rangle=\left\langle F_{i} D_{i}\right\rangle=\left\langle\left[F_{0}+\left(F_{i}-F_{0}+\Delta\right) e^{-\frac{T_{i}}{\tau_{F}}}\right]\right. \\
& \left.\times\left[1+\left(D_{i}-1-F_{i} D_{i}\right) e^{-\frac{T_{i}}{\tau_{D}}}\right]\right\rangle \\
& =F_{0}+\left\langle e^{\left.-\frac{T_{i}}{\tau_{F}}\right\rangle}\left[\left\langle F_{i}\right\rangle-F_{0}+\Delta\right]+\right. \\
& \left\langle e^{-\frac{T_{i}}{\tau_{D}}}\right\rangle F_{0}\left[\left\langle D_{i}\right\rangle-1-\left\langle F_{i} D_{i}\right\rangle\right]+ \\
& \left\langle e^{-T_{i}\left(\frac{1}{\tau_{D}}+\frac{1}{\tau_{F}}\right)}\right\rangle\left[\left(F_{0}-\Delta\right)\left(1-\left\langle D_{i}\right\rangle+\left\langle F_{i} D_{i}\right\rangle\right)+\right. \\
& \left.\left\langle F_{i} D_{i}\right\rangle-\left\langle F_{i}\right\rangle-\left\langle F_{i}^{2} D_{i}\right\rangle\right] .
\end{aligned}
$$

This equation (and all higher-order equations) contain a higher power on the rhs (the term $\left\langle F_{\mathrm{i}}^{2} D_{i}\right\rangle$ ). In other words, the hierarchy of equations for the moments is not closed, and therefore we have to make an approximation. Here we choose a cumulant expansion (Stratonovich, 1967; Gardiner, 1985). Our strategy is to neglect all cumulants higher than of second order, i.e., we make a Gaussian approximation for the joint probability density of $D_{i}$ and $F_{i}$ :

$$
\left\langle\left\langle F_{i}^{n} D_{i}^{m}\right\rangle\right\rangle=0, n+m>2,
$$

where the double brackets indicate the cumulant of the respective quantity. To express the cumulants by moments, one can use a procedure due to van Kampen [see Gardiner (1985), sec. 2.7.1]. Specifically for the product $F_{\mathrm{i}}^{2} D_{i}$, the cumulant reads

$$
\left\langle\left\langle F_{i}^{2} D_{i}\right\rangle\right\rangle=\left\langle F_{i}^{2} D_{i}\right\rangle-\left\langle F_{i}^{2}\right\rangle\left\langle D_{i}\right\rangle+2\left[\left\langle F_{i}\right\rangle^{2}\left\langle D_{i}\right\rangle-\left\langle F_{i}\right\rangle\left\langle F_{i} D_{i}\right\rangle\right] .
$$

Using Equation 25, we thus approximate the moment $\left\langle F_{\mathrm{i}}^{2} D_{i}\right\rangle$ as follows:

$$
\left\langle F_{i}^{2} D_{i}\right\rangle=2\left\langle F_{i}\right\rangle\left\langle F_{i} D_{i}\right\rangle-2\left\langle F_{i}\right\rangle^{2}\left\langle D_{i}\right\rangle+\left\langle D_{i}\right\rangle\left\langle F_{i}^{2}\right\rangle .
$$

Inserting this expression into Equation 24, solving it for the mean $\left\langle F_{\mathrm{i}}^{2} D_{i}\right\rangle$, using Equations 21 and 22, and finally, calculating the different exponentials of the ISI $T_{i}$ by means of Equation 20, we arrive at the approximate formula for the mean value (Eq. 4).

\section{The second moment of the amplitude}

To calculate the variance of the spike train's amplitude, we need to calculate the second moment of $F_{i} D_{i}$, i.e., $\left\langle F_{i}^{2} D_{i}^{2}\right\rangle$. We use again Equation 25 to approximate $\left\langle F_{i}^{2} D_{i}^{2}\right\rangle$ and $\left\langle F_{i} D_{i}^{2}\right\rangle$ by lower-order moments, yielding

$$
\begin{gathered}
\left\langle F_{i}^{2} D_{i}^{2}\right\rangle=\left\langle F_{i}^{2}\right\rangle\left\langle D_{i}^{2}\right\rangle+6\left\langle F_{i}\right\rangle^{2}\left\langle D_{i}\right\rangle^{2}- \\
2\left[\left\langle F_{i}\right\rangle^{2}\left\langle D_{i}^{2}\right\rangle+\left\langle F_{i}^{2}\right\rangle\left\langle D_{i}\right\rangle^{2}-\left\langle D_{i}\right\rangle\left\langle F_{i}^{2} D_{i}\right\rangle+\right. \\
\left.4\left\langle F_{i}\right\rangle\left\langle D_{i}\right\rangle\left\langle F_{i} D_{i}\right\rangle-\left\langle F_{i}\right\rangle\left\langle F_{i} D_{i}^{2}\right\rangle-\left\langle F_{i} D_{i}\right\rangle^{2}\right]
\end{gathered}
$$

and

$$
\left\langle F_{i} D_{i}^{2}\right\rangle=\left\langle F_{i}\right\rangle\left\langle D_{i}^{2}\right\rangle-2\left[\left\langle F_{i}\right\rangle\left\langle D_{i}\right\rangle^{2}-\left\langle D_{i}\right\rangle\left\langle F_{i} D_{i}\right\rangle\right] .
$$

Furthermore, from the stochastic maps, we can obtain equations for the second moments of $F_{i}$ and $D_{i}$, reading

$$
\begin{array}{r}
\left(1-\left\langle e^{-2 \frac{T_{i}}{\tau_{F}}}\right\rangle\right)\left\langle F_{i}^{2}\right\rangle=2 F_{0}\left(\left\langle F_{i}\right\rangle+\Delta-F_{0}\right)\left\langle e^{-\frac{T_{i}}{\tau_{F}}}\right\rangle+F_{0}^{2}+ \\
{\left[2\left\langle F_{i}\right\rangle\left(\Delta-F_{0}\right)+\left(\Delta-F_{0}\right)^{2}\right]\left\langle e^{-2 \frac{T_{i}}{\tau_{F}}}\right\rangle}
\end{array}
$$

$$
\begin{aligned}
\left(1-\left\langle e^{-2 \frac{T_{i}}{\tau_{D}}}\right\rangle\right)\left(1-2\left\langle D_{i}\right\rangle+\left\langle D_{i}^{2}\right\rangle\right) & =\left(\left\langle F_{i}^{2} D_{i}^{2}\right\rangle-2\left\langle F_{i} D_{i}^{2}\right\rangle\right. \\
& \left.+2\left\langle F_{i} D_{i}\right\rangle\right)\left\langle e^{-2 \frac{T_{i}}{\tau_{D}}}\right\rangle .
\end{aligned}
$$

The resulting five linear equations (Eqs. 27-31) uniquely determine the unknown moments $\left\langle F_{i}^{2}\right\rangle,\left\langle D_{i}^{2}\right\rangle,\left\langle F_{i} D_{i}^{2}\right\rangle,\left\langle F_{\mathrm{i}}^{2} D_{i}\right\rangle$, and $\left\langle F_{i}^{2} D_{i}^{2}\right\rangle$. The resulting expression for the second moment of $\left\langle F_{i}^{2} D_{i}^{2}\right\rangle$ is too cumbersome to be presented here. It can, however, be readily obtained by solving the above equations using computer algebra software. Comparisons to simulation data show excellent agreement as long as the condition in Equation 23 is not violated (data not shown).

\section{Power spectrum of the amplitude-modulated spike train}

Here we calculate an approximation for the power spectrum $S_{x x}$ at a single synapse $x_{j}(t)$ (the index $j$ is omitted again in the following). If the set of input spike times $\left\{t_{i}\right\}$ corresponds to a Poissonian point process, we can write the power spectrum of a single synaptic input as follows (using the index $k$ in place of $i$ to avoid confusion with the imaginary $i$ ):

$$
\begin{gathered}
S_{x x}=r\left\langle\sum_{l=-\infty}^{\infty} A_{k} A_{k+l} e^{2 \pi i f\left(t_{k+l}-t_{k}\right)}\right\rangle \\
=\left\langle A_{k}\right\rangle^{2} r+ \\
r\left\langle\sum_{l=-\infty}^{\infty}\left(A_{k} A_{k+1}-\left\langle A_{k}\right\rangle\left\langle A_{k+1}\right\rangle\right) e^{2 \pi i f\left(t_{k+1}-t_{k}\right)}\right\rangle,
\end{gathered}
$$

where we used the abbreviation for the synaptic amplitude $A_{k}=$ $F_{k} D_{k}$ ( $k$ refers to the spike time, not to the synapse). In deriving Equation 32, we assume that the number of spikes divided by the time window gives the spike rate $r$ in the limit. The triple correlations in the last line are hard to calculate. For low input rate, though, only correlations between $A_{i+1}$ and the previous ISI $T_{i}$ dominate. This assumption leads to the following expression for the spectrum:

$S_{x x} \approx r\left\langle A_{k}^{2}\right\rangle+2 r\left\langle A_{k}\right\rangle\left(\Re\left\langle A_{k+1} e^{2 \pi i f T_{k}}\right\rangle-\frac{r}{2 \pi f} \mathfrak{J}\left\langle A_{k+1} e^{2 \pi i f T_{k}}\right\rangle\right)$.

Using the maps in Equations 18 and 19, the synaptic amplitude at the $k+1$ spike can be expressed by the values of the synaptic variables at the $k$ th spike:

$$
\begin{aligned}
A_{k+1}=F_{k+1} D_{k+1}= & \left(F_{0}+\left(F_{k}-F_{0}+\Delta\right) e^{-T_{k} / \tau_{F}}\right) \\
& \times\left(1+\left(D_{k}-1-F_{k} D_{k}\right) e^{-T_{k} / \tau_{D}}\right) .
\end{aligned}
$$

After multiplying the last equation with $\mathrm{e}^{2 \pi i f T_{k}}$, we can separately average all terms consisting of products of the synaptic variables and all exponentials containing the subsequent ISI (which is independent of $F_{k}$ and $D_{k}$ and their products). With $S_{X X}=N S_{x x}$, for statistically independent inputs [see also Lindner (2006) for a recent discussion], we obtain a lengthy expression for the power spectrum with Lorentzian correction terms, the prefactors of which contain the averages $\left\langle D_{k}\right\rangle,\left\langle F_{k}\right\rangle,\left\langle F_{k} D_{k}\right\rangle$, and $\left\langle F_{k}^{2} D_{k}\right\rangle$ (see previous sections):

$$
\begin{aligned}
S_{x x}= & N r\left\langle A_{k}^{2}\right\rangle+2 N r^{2}\left\langle A_{k}\right\rangle \times \\
& {\left[\frac{\left(\left\langle F_{k}\right\rangle+\Delta-F_{0}\right) \tau_{F}}{\left(1+r \pi_{F}\right)^{2}+\left(2 \pi f \tau_{F}\right)^{2}}+\frac{F_{0} \tau_{D}\left(\left\langle D_{k}\right\rangle-\left\langle F_{k} D_{k}\right\rangle-1\right)}{\left(1+r \tau_{D}\right)^{2}+\left(2 \pi f \tau_{D}\right)^{2}}\right.} \\
& \left.+\frac{\left\langle F_{k} D_{k}\right\rangle\left(1+F_{0}-\Delta\right)-\left\langle F_{k}\right\rangle+\left(F_{0}-\Delta\right)\left(1-\left\langle D_{k}\right\rangle\right)-\left\langle F_{k}^{2} D_{k}\right\rangle}{(1+r \tilde{\tau})^{2}+(2 \pi f \tilde{\tau})^{2}}\right] .
\end{aligned}
$$


This expression and Equation 6 have been used in Figure $1 D$ to calculate the theoretical curves of the conductance spectrum. If we set $r=0$ in the square bracket in Equation 35, we obtain the more instructive expression given in the main text in Equation 7 in which the Lorentzian correction terms are given in a simple way by the synaptic parameters. At low rate $(r=1 \mathrm{~Hz}$ in Fig. $1 D)$, Equations 35 and 7 yield very similar spectra. Quantitative deviations between these approximations have been observed for the FDR at $r=10 \mathrm{~Hz}$.

\section{Separating FDR and DDR}

Here, we sketch out the derivation of Equation 17. We first investigate whether the mean synaptic amplitude increases or decreases by increasing the input's firing rate. This will depend on the rate itself (the amplitude can go through a maximum as a function of rate) (see Fig. $1 B$ ), and to be definite, we study the sign of the mean's derivative at zero rate. Taking the derivative of Equation 4 with respect to $r$ at $r=0$ yields

$$
\left.\frac{d\left\langle F_{i} D_{i}\right\rangle}{d r}\right|_{r=0}=-F_{0}^{2} \tau_{D}+\Delta \tau_{F}\left(1-\frac{F_{0}}{1+\tau_{F} / \tau_{D}}\right),
$$

and the condition that this is positive (the synapse is dominated by facilitation) leads to Equation 17.

Second, we may ask under which condition the modification of the power spectrum by the three Lorentzian terms is positive at zero frequency and for low input rate. Subtracting from the power spectrum (Eq. 7) its high-frequency limit $r N\left\langle A_{i}^{2}\right\rangle$, we find that the resulting difference is positive at zero frequency if

$$
\Delta \tau_{F}-F_{0}^{2} \tau_{D}-\Delta F_{0} \tilde{\tau}>0
$$

which indeed corresponds to Equation 17. Hence, dominating facilitation is necessarily associated with increase of power at low frequencies.

\section{References}

Abbott LF, Regehr WG (2004) Synaptic computation. Nature 431:796-803. Abbott LF, Varela JA, Sen K, Nelson SB (1997) Synaptic depression and cortical gain control. Science 275:220-224.

Bastian J, Chacron MJ, Maler L (2004) Plastic and nonplastic pyramidal cells perform unique roles in a network capable of adaptive redundancy reduction. Neuron 41:767-779.

Chance FS, Abbott LF, Reyes AD (2002) Gain modulation from background synaptic input. Neuron 35:773-782.

Dayan P, Abbott LF (2001) Theoretical neuroscience. Cambridge, MA: MIT.

Destexhe A, Marder E (2004) Plasticity in single neurons and circuit computations. Nature 431:789-795.

Dittman JS, Kreitzer AC, Regehr WG (2000) Interplay between facilitation, depression, and residual calcium at three presynaptic terminals. J Neurosci 20:1374-1385.

Doiron B, Longtin A, Berman N, Maler L (2001) Subtractive and divisive inhibition: effect of voltage-dependent inhibitory conductances and noise. Neural Comput 13:227-248.

Fortune ES, Rose GJ (2001) Short-term synaptic plasticity as a temporal filter. Trends Neurosci 24:381-385.

French AS, Holden AV, Stein RB (1972) The estimation of the frequency response function of a mechanoreceptor. Kybernetik 11:15-23.
Fuhrmann G, Markram H, Tsodyks M (2002) Spike frequency adaptation and neocortical rhythms. J Neurophysiol 88:761-770.

Gabbiani F, Koch C (1998) Principles of spike train analysis. In: Methods in neuronal modeling: from ions to networks (Koch C, Segev I, eds), pp 313-360. Cambridge, MA: MIT.

Gammaitoni L, Hänggi P, Jung P, Marchesoni F (1998) Stochastic resonance. Rev Mod Phys 70:223-287.

Gardiner CW (1985) Handbook of stochastic methods. Berlin: Springer.

Goldman MS, Maldonado P, Abbott LF (2002) Redundancy reduction and sustained firing with stochastic depressing synapses. J Neurosci 22:584-591.

Jacobson GA, Diba K, Yaron-Jakoubovitch A, Oz Y, Koch C, Segev I, Yarom Y (2005) Subthreshold voltage noise of rat neocortical pyramidal neurones. J Physiol 564:145-160.

Klyachko VA, Stevens CF (2006) Excitatory and feed-forward inhibitory hippocampal synapses work synergistically as an adaptive filter of natural spike trains. PLoS Biol 4:e207.

Knight BW (1972) Dynamics of encoding in a population of neurons. J Gen Physiol 59:734-766.

Kuhn A, Aertsen A, Rotter S (2004) Neuronal integration of synaptic input in the fluctuation-driven regime. J Neurosci 24:2345-2356.

Le Masson G, Renaud-Le Masson S, Debay D, Bal T (2002) Feedback inhibition controls spike transfer in hybrid thalamic circuits. Nature 417:854-858.

Lewis JE, Maler L (2002) Dynamics of electrosensory feedback: short-term plasticity and inhibition in a parallel fiber pathway. J Neurophysiol 88:1695-1706.

Lewis JE, Maler L (2004) Synaptic dynamics on different time scales in a parallel fiber feedback pathway of the weakly electric fish. J Neurophysiol 91:1064-1070.

Lindner B (2006) Superposition of many independent spike trains is generally not a Poisson process. Phys Rev E 73:022901.

Lisman JE (1997) Bursts as a unit of neural information: making unreliable synapses reliable. Trends Neurosci 20:38-43.

Longtin A (1993) Stochastic resonance in neuron models. J Stat Phys 70:309-327.

Markram H, Tsodyks MV (1996) Redistribution of synaptic efficacy between neocortical pyramidal neurons. Nature 382:807-810.

McNamara B, Wiesenfeld K (1989) Theory of stochastic resonance. Phys Rev A 39:4854-4869.

Mochida S, Few AP, Scheuer T, Catterall WA (2008) Regulation of presynaptic Ca v2.1 channels by $\mathrm{Ca} 2+$ sensor proteins mediates short-term synaptic plasticity. Neuron 57:210-216.

Mongillo G, Barak O, Tsodyks M (2008) Synaptic theory of working memory. Science 319:1543-1546.

Morrison A, Diesmann M, Gerstner W (2008) Phenomenological models of synaptic plasticity based on spike timing. Biol Cybern 98:459-478.

Oswald AM, Chacron MJ, Doiron B, Bastian J, Maler L (2004) Parallel processing of sensory input by bursts and isolated spikes. J Neurosci 24:4351-4362.

Puccini GD, Sanchez-Vives MV, Compte A (2007) Integrated mechanisms of anticipation and rate-of-change computations in cortical circuits. PLoS Comput Biol 3:e82.

Rieke F, Warland D, de Ruyter van Steveninck R, Bialek W (1996) Spikes: exploring the neural code. Cambridge, MA: MIT.

Stein RB, Gossen ER, Jones KE (2005) Neuronal variability: noise or part of the signal? Nat Rev Neurosci 6:389-397.

Stratonovich RL (1967) Topics in the theory of random noise. New York: Gordon and Breach.

Tsodyks MV, Markram H (1997) The neural code between neocortical pyramidal neurons depends on neurotransmitter release probability. Proc Natl Acad Sci U S A 94:719-723.

Zucker RS, Regehr WG (2002) Short-term synaptic plasticity. Annu Rev Physiol 64:355-405. 